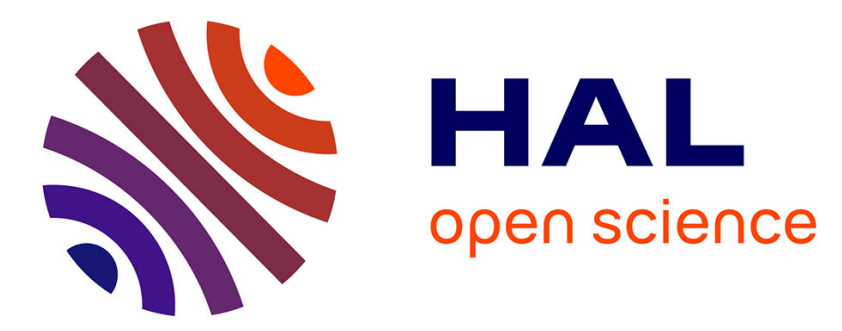

\title{
Dynamic crack propagation with a variational phase-field model: limiting speed, crack branching and velocity-toughening mechanisms
}

Jeremy Bleyer, Clément Roux-Langlois, Jean-François Molinari

\section{- To cite this version:}

Jeremy Bleyer, Clément Roux-Langlois, Jean-François Molinari. Dynamic crack propagation with a variational phase-field model: limiting speed, crack branching and velocity-toughening mechanisms. International Journal of Fracture, 2017, 204 (1), pp.79-100. 10.1007/s10704-016-0163-1. hal-01390233

\section{HAL Id: hal-01390233 \\ https://hal-enpc.archives-ouvertes.fr/hal-01390233}

Submitted on 1 Nov 2016

HAL is a multi-disciplinary open access archive for the deposit and dissemination of scientific research documents, whether they are published or not. The documents may come from teaching and research institutions in France or abroad, or from public or private research centers.
L'archive ouverte pluridisciplinaire $\mathbf{H A L}$, est destinée au dépôt et à la diffusion de documents scientifiques de niveau recherche, publiés ou non, émanant des établissements d'enseignement et de recherche français ou étrangers, des laboratoires publics ou privés. 


\title{
Dynamic crack propagation with a variational phase-field model: limiting speed, crack branching and velocity-toughening mechanisms
}

\author{
Jérémy Bleyer* • Clément Roux-Langlois • Jean-François Molinari
}

Received: date / Accepted: date

The final publication is available at:

link.springer.com/article/10.1007/s10704-016-0163-1

Abstract We address the simulation of dynamic crack propagation in brittle materials using a regularized phasefield description, which can also be interpreted as a damage-gradient model. Benefiting from a variational framework, the dynamic evolution of the mechanical fields are obtained as a succession of energy minimizations. We investigate the capacity of such a simple model to reproduce specific experimental features of dynamic in-plane fracture. These include the crack branching phenomenon as well as the existence of a limiting crack velocity below the Rayleigh wave speed for mode I propagation. Numerical results show that, when a crack accelerates, the damaged band tends to widen in a direction perpendicular to the propagation direction, before forming two distinct macroscopic branches. This transition from a single crack propagation to a branched configuration is described by a well-defined master-curve of the apparent fracture energy $\Gamma$ as an increasing function of the crack velocity. This $\Gamma(v)$ relationship can be associated, from a macroscopic point of view, with the well-known velocity-toughening mechanism. These results also support the existence of a critical value of the

J. Bleyer · C. Roux-Langlois · J-F. Molinari

Ecole Polytechnique Fédérale de Lausanne

Department of Civil Engineering

Department of Materials Science

1015 Lausanne, Switzerland Tel.: +41 216932413

* Corresponding author: J. Bleyer

E-mail: jeremy.bleyer@\{epfl.ch,enpc.fr\}

C. Roux-Langlois

University of Rennes 1

Institute of physics of Rennes

Mechanics and Glasses Department, UMR UR1-CNRS 6251,

Campus de Beaulieu, 35042 Rennes Cedex, France energy release rate associated with branching: a critical value of approximately $2 G_{c}$ is observed i.e. the fracture energy contribution of two crack tips. Finally, our work demonstrates the efficiency of the phase-field approach to simulate crack propagation dynamics interacting with heterogeneities, revealing the complex interplay between heterogeneity patterns and branching mechanisms.

Keywords Dynamic fracture - Crack branching . Brittle materials · Phase-field model · Damage-gradient model

\section{Introduction}

Understanding the various mechanisms governing the dynamic propagation of a crack in a brittle medium is still a challenge. The difficulty lies in the strong interaction between stress concentrations at the crack tip, various non-linear phenomena occurring in the process zone, material heterogeneities at potentially different scales and dynamic stress redistribution due to waves emitted by the moving crack tip and reflections at the boundaries. For a single propagating crack, linear fracture mechanics relies on the balance between a crack driving force, the dynamic energy release rate, and a crack resisting force given by the fracture energy, which is assumed to be a material property. It also predicts that the Rayleigh wave speed $c_{R}$ is the limiting velocity of a mode I propagating crack [63]. However, various experimental results have shown that this simple picture of dynamic brittle fracture is far from being complete. In particular, the existence of limiting velocities below $c_{R}$, microscopic and macroscopic crack branching phenomena and a dependence of the fracture energy on the crack velocity have been observed experimentally. 


\subsection{Limiting speed and dynamic instabilities}

In the absence of branching or before its occurrence, experiments have reported the following features of dynamic crack propagation $[56,14]$ :

- cracks accelerate to a constant limiting velocity;

- this limiting velocity depends on the experimental setup for a given material;

- the stress intensity factor and surface roughness increase during the constant velocity phase.

Although the limiting velocity may depend on loading, boundary conditions or geometry, it has been measured to be in the range of $0.5-0.65 c_{R}$ for glass, $0.6-$ $0.7 c_{R}$ for PMMA and $0.35-0.45 c_{R}$ for Homalite-100 [26].

Experiments have also reported that branching can be suppressed by constraining the crack path to a weaker interface or by drilling an array of holes ahead of the crack [66]. In these cases, crack velocities can reach up to $90 \%$ of the Rayleigh wave speed.

In [23], Gao proposed a principle maximizing the fracture energy flux $\left(\dot{\Gamma}_{a}=G\left(v_{a}\right) \cdot v_{a}\right.$ with $G$ the dynamic energy release rate and $v_{a}$ the apparent crack velocity) i.e. the energy being absorbed into the fracture process per unit time per unit length of the crack front. A wavy-crack model is introduced in which the crack tip follows a wavy path corresponding to a local (microscopic) crack tip speed which may be very high (close to $c_{R}$ ) whereas its apparent (macroscopic) speed $v_{a}$ is only a fraction of $c_{R}$.

Experiments have evidenced different regimes for crack propagation controlled by dynamic instabilities [19]. At low velocities, the crack surface appears almost flat (mirror regime), velocity oscillations and an increase of surface roughening in the form of conic marks start to appear at higher velocities (mist regime), at even higher velocities microbranching and important surface roughening occur (hackle regime). A coalescence of microcracks in the crack process zone has also been proposed as a potential mechanism triggering this instability [55]. Recent experiments over a wide range of crack velocities $[25,16]$ have confirmed that these instabilities are at the origin of the limiting speed: before attaining $c_{R}$, such instabilities prevent the crack to further accelerate and the picture of a single propagating crack is not appropriate anymore.

\subsection{Branching criterion}

Yoffe showed that the circumferential stress at the crack tip reaches a maximum at a $60^{\circ}$ angle when the crack tip speed exceeds $0.6 c_{s}$ [70]. Unfortunately, this argument cannot explain the critical velocities and branching angles observed in the experiments.

Crack branching can be viewed as a consequence of an excess of available energy flowing to the crack tip which cannot be dissipated by a single crack propagation. Indeed, stress intensity factors (SIF) and energy release rates (ERR) increase prior to branching then drop since energy is now used to propagate two branches (explaining the smooth aspect of the crack surface just after branching). This suggests the existence of a critical value of SIF or ERR related to branching $[59,54]$. Because of the dependence of SIF on crack velocity [22], this critical value could also be related to a critical crack tip velocity. However, there is no universal relation between dynamic SIF and crack tip velocity [9]. Besides, experiments indicate that branches propagate at roughly the same speed as the main crack before branching, therefore weakening the assumption that the crack tip velocity is the relevant criterion for crack branching [55].

1.3 Velocity-toughening mechanism of the fracture energy

Experiments on PMMA for a strip geometry [60] (similar to the one considered later in this paper) reported a strong increase of the apparent fracture energy with the crack velocity, ranging from approximately 1,000 J/m $\mathrm{m}^{2}$ for $v=0.2 c_{R}$ up to approximately $8,000 \mathrm{~J} / \mathrm{m}^{2}$ for $0.68 c_{R}$. However, this strong increase is attributed to an important increase of relative surface area created by a crack advance through branching instabilities. The amount of fracture surface is shown to be linearly related to the energy flux into the crack tip so that the local fracture energy can still be considered as constant (equal to $1,100 \mathrm{~J} / \mathrm{m}^{2}$ ) for $v \geq v_{c}=0.35 c_{R}$, where $v_{c}$ was identified as the critical velocity associated with the microbranching instability.

In contrast to these dynamic measurements of PMMA fracture energy, quasi-static experiments usually report values in the range of $300-400 \mathrm{~J} / \mathrm{m}^{2}$. In $[25,16]$, a wedgesplitting experimental setup enabled to measure fracture energy at very low velocities. Between $0.11 c_{R}$ and $0.18 c_{R}$, the fracture energy increases abruptly between $400 \mathrm{~J} / \mathrm{m}^{2}$ and $1,200 \mathrm{~J} / \mathrm{m}^{2}$ i.e. from a value consistent with quasi-static measurements to a value consistent with the results of [60]. Interestingly, in the slow crack 
regime, a single propagating front is observed whereas, for $v \geq v_{a}=0.18 c_{R}$ nucleations of microdefects propagating at $v_{a}$ ahead of the main crack are observed. The collective dynamics of the main crack and microdefects yield an apparent crack velocity higher than $v_{a}$.

These different results highlight the fact that the dynamic behaviour of crack propagation in PMMA is extremely complex, resulting from microdefects nucleation, microbranching instabilities, an increase of relative surface area and maybe a thickening of the damage zone. Experiments have also shown that microcracking and surface roughening are complex 3D phenomena [24] and that geometrical non-linearities at the crack tip may have to be taken into account to overcome LEFM deficiencies [39].

1.4 Finite element models of dynamic crack propagation

Unfortunately, an exhaustive modeling of all the previously mentioned phenomena would certainly require very fine $3 \mathrm{D}$ computations in a heterogeneous medium, which are still out of reach. Different classes of models have been proposed to simulate some of these dynamic crack features in brittle materials at a macro-scale.

Cohesive zone models $[5,17,69]$ represent the crack propagation process by considering a potential opening between two bulk elements. The cohesive law defines the constitutive relation between the surface traction and the relative opening displacement. By constraining the crack propagation along the element edges, this approach suffers from a mesh dependency at small scales but seems able to capture some features of crack branching patterns $[69,18,73]$ and the existence of a limiting velocity.

The extended finite element method (XFEM) enriches the finite element interpolation by adding either singularities or strong discontinuities for elements cut by the crack [46]. The level-set is an efficient method to describe the crack path, and is now widely used [62]. Although XFEM enables to have a discrete representation of the crack, one main drawback is that additional branching criteria and velocity toughening models are needed as input of the crack propagation algorithm to be able to obtain branched configurations $[6,68]$.

Non-local approaches regroup a wide range of models which represent the discrete crack by a continuous damage field and a regularization length to remove any mesh dependency. One can mention the non-local integral approach $[53,31,67]$, gradient-enhanced models [51], eigenerosion [49], peridynamics [7], thick-level sets [47] and phase-field approaches, which will be described later in more details. Generally, such non-local approaches do not require additional criteria to obtain branched patterns $[34,27,29,30]$.

In [28], the Karma-Kessler-Levine phase-field model [33] is used to study crack branching in 3D and its relation to fractographic patterns induced by the instability. Depending on the simulation parameters, 2D crack fronts or complex 3D patterns have been obtained. It is highlighted that crack velocities for $3 \mathrm{D}$ patterns are usually smaller than those observed for $2 \mathrm{D}$ patterns and are also associated with a higher total fracture surface.

Dynamic crack propagation using the phase-field approach has also been investigated in $[8,30,36,37]$. In some cases, branching angles and crack velocities have been reported to be close to experimental observations whereas strange patterns have also been observed in other situations. In particular, the choice of the tensioncompression splitting has been shown to have an important influence on the observed pattern [37]. In [30], crack branching is associated with a critical value of the crack surface velocity.

In [7], mode I crack branching has been investigated using a peridynamic formulation of brittle fracture. Results have shown a dependence on loading conditions and sample geometry of branching angles and crack tip velocities. The authors proposed a stress-wave pile-up mechanism and damage spreading to the crack faces as the origin of crack branching.

It is to be noted that peridynamics rely on the same mechanical ingredients as phase-field modeling of brittle fracture, namely a linear elastic continuum model, a dissipation mechanism controlled by a material fracture energy and an intrinsic regularization length. It is therefore quite interesting that both approaches are able to reproduce characteristic aspects of crack branching without any additional criterion.

\subsection{Objectives and organization of the manuscript}

The purpose of the present work is to address the simulation of dynamic crack propagation using variational phase-field models. These models have emerged in the last decade as a promising tool for brittle fracture simulation as they do not require any a priori knowledge of the crack path or topology while being much less sensitive to the mesh discretization compared to cohesive zone models for instance. A large number of previous works on phase-field approaches for dynamic fracture 
mostly concentrated on numerical issues and qualitative aspects of the branched patterns. To our knowledge, a close inspection of the physical mechanisms at the origin of dynamic crack propagation has not been realized yet in the phase-field framework, especially regarding the conditions leading to crack branching. We aim here at demonstrating that such models can reproduce experimentally observed features of brittle dynamic fracture such as the existence of a limiting velocity and an increase of apparent fracture energy with the velocity.

In section 2 , the general formulation of the phasefield model used in this work is briefly recalled. Constitutive modeling choices as well as numerical aspects are also discussed. In section 3 , the numerical simulation of dynamic crack propagation in a pre-strained plate is investigated and the occurrence of crack branching and the existence of a velocity-toughening mechanism are more particularly examined. Finally, section 4 is devoted to the simulation of crack propagation in a heterogeneous medium. Situations in which the crack is constrained to propagate along a weak plane as well as propagation in presence of distant heterogeneities are investigated.

\section{Variational phase-field models of brittle fracture}

The phase-field models proposed in the literature as regularized models of brittle fracture can be split in two categories. The first category is based on a GinzburgLandau phase transformation evolution equation [33, $27,28]$. Although this first approach enabled to produce interesting results regarding crack branching, mixedmode instabilities and surface roughening, some issues concerning the evolution of the phase field away from the crack tip, even if the sample has been brought to equilibrium, have been raised in [13]. Besides, this formulation seems more distant to Griffith's theory of fracture although some physical parameters can be related to a fracture energy and a regularization length.

The second category of phase-field models has been developed as a regularization of free discontinuity problems $[2,11]$ arising in the variational approach to fracture proposed in [21]. In this case, a continuous function $d$ varying between 0 (sound material) and 1 (fully cracked material) is introduced to obtain a smooth formulation of the Griffith fracture functional by a crack density function depending on $d$ and $\underline{\nabla d}$. This functional is parametrized by the Griffith fracture energy $G_{c}$ and an internal length scale $l_{0}$, which is related to the distance over which the phase field varies from 0 to 1 in a localized damaged band. The majority of the phase-field models adopted in the literature are based on this formulation, either in a rate-independent form $[44,13,8]$ or in a rate-dependent form including viscous dissipation of crack propagation as a numerical regularization of the time evolution problem $[45,29,30]$.

Continuum descriptions of cohesive fractures have also been proposed using the phase-field approach [43, 65], continuum damage-gradient [41] or the thick-level set (TLS) method [47], see also [15] for a comparison between the TLS and the phase-field approach. Recent works also proposed to extend the phase-field approach to the case of ductile fracture in an elastoplastic-damageable formulation [1].

Phase-field models based on a regularization of the variational approach to fracture share strong links with continuum damage-gradient models [52]. In fact, the phase-field model based on [11] belongs to the general class of gradient damage models for a specific choice of damage constitutive laws. These damage-gradient models have been recently extended to the dynamic propagation in $[36,37]$. Due to the variational formulation of these models and their generality, we will adopt this point of view in the remainder of this work.

Finally, let us mention that, for crack-like localized solutions, quasi-static damage-gradient models converge to the Griffith theory of brittle fracture when the internal length scale $l_{0}$ is much smaller than the characteristic length $L$ of the structure [61]. Although no formal proof has been published yet concerning the dynamic case, it seems reasonable to expect that such a result will still hold in this case.

\subsection{Formulation of the variational approach to dynamic fracture}

We briefly recall here the general formulation of the variational phase-field or gradient damage model of dynamic fracture. We invite the reader to refer to [37] and to references mentioned therein for more details on the construction of such models.

In the context of isotropic damage models, a continuous scalar field $d$, taking values in the $[0 ; 1]$ interval, is introduced as a smooth representation of a transition between intact material $(d=0)$ and a fully cracked material $(d=1)$ (see Figure 1$)$. Denoting by $\underline{u}$ a kinematically admissible displacement field and by $\underline{\dot{u}}$ its associated velocity, the variational gradient damage model relies on the definition of the following energies:

- the elastic strain energy:

$$
E_{e l}(\underline{u}, d)=\int_{\Omega} \psi(\underline{\underline{\varepsilon}}, d) d \Omega
$$




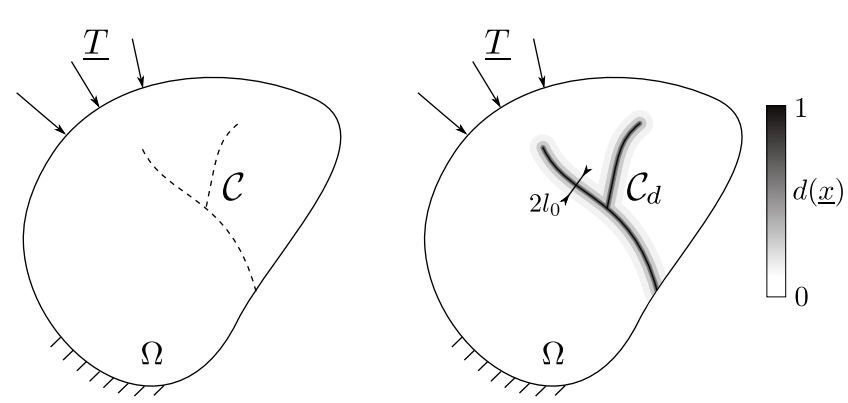

Fig. 1 Continuum regularization of a possibly branched crack $\mathcal{C}$ by a continuous phase field $d(x) \in[0 ; 1]$ in a continuum $\Omega$ subjected to imposed displacement and tractions $\underline{T}$ at its boundary. The regularized crack corresponds to the domain $\mathcal{C}_{d}$ for which $d>0$, its typical width is related to $l_{0}$.

where $\Omega$ represents the continuum (Fig. 1), $\underline{\underline{\varepsilon}}=$ $\frac{1}{2}\left(\underline{\underline{\nabla u}}+\underline{\underline{\nabla u}}{ }^{\mathrm{T}}\right)$ is the linearized strain tensor associated with $\underline{\underline{u}}, \psi$ being the elastic potential depending on $d$;

- the kinetic energy:

$$
E_{k i n}(\underline{\dot{u}})=\int_{\Omega} \frac{1}{2} \rho \underline{\dot{u}} \cdot \underline{\dot{u}} d \Omega
$$

where $\rho$ is the material density;

- the non-local damage or fracture energy:

$$
E_{f r a c}(d)=\int_{\Omega}\left(w(d)+W_{0} l_{0}^{2} \underline{\nabla d} \cdot \underline{\nabla d}\right) d \Omega
$$

where $w(d)$ is the local damage energy density, $W_{0}$ is homogeneous to an energy density and $l_{0}$ is homogeneous to a distance.

Finally, an action-integral over a time interval $I=$ $\left[t_{1}, t_{2}\right]$ is introduced as follows:

$\mathcal{A}=\int_{I}\left(E_{e l}(\underline{u}, d)+E_{f r a c}(d)-E_{k i n}(\underline{\dot{u}})-W_{e x t}(\underline{u})\right) d t$

where $W_{\text {ext }}(\underline{u})$ represents the work done by the external forces on the displacement field $\underline{u}$.

The variational formulation of the gradient damage model, giving the space-time evolution of the mechanical fields $(\underline{u}, d)$, is then obtained by assuming that:

- the damage variable evolution is irreversible: $\dot{d} \geq 0$ for all $t$;

- the variation action-integral is positive with respect to arbitrary variations of admissible displacements and damage evolution;

- energy is dissipated only via the fracture energy $E_{\text {frac }}(d)$.
The derivation of these principles, especially the second one, shows that the elastodynamic equilibrium equation is obtained for a damage-dependent stress state $\underline{\underline{\sigma}}=$ $\partial_{\underline{\underline{\varepsilon}}} \psi(\underline{\underline{\varepsilon}}, d)$ :

$\operatorname{div} \underline{\underline{\sigma}}+\underline{f}=\rho \underline{\ddot{u}}$

with appropriate boundary conditions and where $\underline{f}$ is a given body force density.

Besides, the variation of (4) in terms of admissible damage evolution results in the total energy satisfying a minimum principle constrained by the irreversibility condition of damage evolution:

$$
E_{e l}(\underline{u}, d)+E_{f r a c}(d) \leq E_{e l}(\underline{u}, \widehat{d})+E_{f r a c}(\widehat{d}) \quad \forall \widehat{d} \in \mathcal{D}(d)
$$

where $\mathcal{D}(d)$ is the admissible space for damage variations from a given damage state $0 \leq d \leq 1$ defined by:

$\mathcal{D}(d)=\{\widehat{d}$ s.t. $0 \leq d \leq \widehat{d} \leq 1\}$

In practice, the wave equation (5) and the damage minimum principle (6) are solved numerically at every time step.

\subsection{Constitutive modeling choices}

The previous formulation is very general and different damage models can be obtained depending on the choice of the damageable elastic energy density $\psi(\underline{\underline{\varepsilon}}, d)$ and the local damage density $w(d)$.

For the elastic strain energy density, we chose the classical form [3]:

$$
\begin{aligned}
\psi(\underline{\underline{\varepsilon}}, d) & =(1-d)^{2} \psi^{+}(\underline{\underline{\varepsilon}})+\psi^{-}(\underline{\underline{\varepsilon}}) \\
\psi^{+}(\underline{\underline{\varepsilon}}) & =\frac{\kappa}{2}\langle\operatorname{tr} \underline{\underline{\varepsilon}}\rangle_{+}^{2}+\mu \underline{\underline{\varepsilon}}^{d}: \underline{\underline{\varepsilon}}^{d} \\
\psi^{-}(\underline{\underline{\varepsilon}}) & =\frac{\kappa}{2}\langle\operatorname{tr} \underline{\underline{\varepsilon}}\rangle_{-}^{2}
\end{aligned}
$$

where $\kappa$ and $\mu$ are the compressibility and shear elastic moduli, $\underline{\underline{\varepsilon}}^{d}$ is the deviatoric strain tensor and $\langle\star\rangle_{ \pm}=$ $(\star \pm|\star|) / 2$ denotes the positive (resp. negative) part of $\star$.

This model enables to distinguish between tension and compression, the damage degradation impacting the deviatoric strain as well as the positive part of the volumetric strain, while the negative part of the volumetric strain is not impacted. This is not the only choice for tension-compression splitting, see [37] for an extensive discussion on this subject. For the problem considered in this paper, the impact of the splitting 


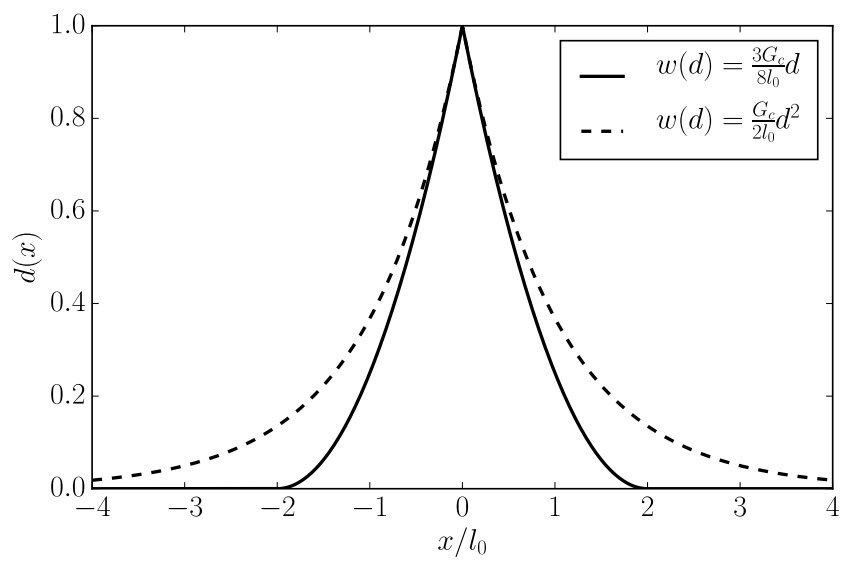

Fig. 2 One dimensional localized solution of an infinite bar in traction for the different damage laws. The total dissipated energy for this test case corresponds exactly to $G_{c}$. For $w(d) \propto d(12)$, the damage profile is $d=\left(1-|x| /\left(2 l_{0}\right)\right)^{2}$ for $|x| \leq 2 l_{0}$ and $d=0$ for $|x| \geq 2 l_{0}$. For $w(d) \propto d^{2}(11)$, the damage profile is an exponential with infinite support $d=\exp \left(-|x| / l_{0}\right)$.

choice is limited as compression stress waves reflected at the boundaries are generated only by the crack tip advance and are of small amplitude.

As regards the choice of the damage energy density, the most widely used model $[11,44,29,8,15]$ is given by $^{1}$ :

$w(d)+W_{0} l_{0}^{2} \underline{\nabla d} \cdot \underline{\nabla d}=\frac{G_{c}}{2 l_{0}}\left(d^{2}+l_{0}^{2} \underline{\nabla d} \cdot \underline{\nabla d}\right)$

where $G_{c}$ is the Griffith fracture energy.

Although appealing from a numerical point of view due to its purely quadratic nature, this model predicts no elastic domain in which the material behaves elastically with zero damage [52]. Therefore, when comparing the macroscopic total dissipated energy with respect to a crack length evolution, a non-negligible part of this dissipated energy actually comes from small damaging of the bulk domain due to the applied stress state. This pre-damaging of the surrounding bulk domain will also tend to reduce the values of the different wave speeds compared to a non-damaged domain. Besides, the localized 1D solution for $d$ of a bar in tension corresponds to an exponentially decreasing function with infinite support (Fig. 2).

For these different reasons, we chose to use the following damage energy density proposed in [52]:

$w(d)+W_{0} l_{0}^{2} \underline{\nabla d} \cdot \underline{\nabla d}=\frac{3 G_{c}}{8 l_{0}}\left(d+l_{0}^{2} \underline{\nabla d} \cdot \underline{\nabla d}\right)$

\footnotetext{
1 with sometimes a non-essential rescaling of the internal
} length $l_{0}$
The absence of a square exponent in the damage variable leads to a pure elastic phase associated with a critical stress $\sigma_{c}=\sqrt{3 G_{c} E /\left(8 l_{0}\right)}$ for a $1 \mathrm{D}$ homogeneous traction test. In this case, the $1 \mathrm{D}$ profile corresponds to a portion of parabola with an exactly zero damage state for $|x| \geq 2 l_{0}$ (Figure 2).

Let us also mention that the normalization of both models is chosen so that, when considering the localized damage solution for a 1D bar in traction, the surface fracture energy corresponds exactly to $G_{c}$.

\subsection{Numerical aspects}

The numerical code used in this work is inspired from the open-source implementation of the dynamic damagegradient model [38] based on the FEniCS project for automated resolution of PDE's [40] and the PETSc library [4].

Classical linear finite element interpolations on triangles are used for the displacement, velocity, acceleration and damage fields. As regards temporal discretization, an explicit Newmark scheme has been chosen for the update of accelerations, velocities and displacements. The damage problem is formulated as a bound-constrained quadratic optimization problem to ensure damage irreversibility. More details on the numerical implementation can be found in [37].

The mesh size has usually been taken approximately 4-5 times smaller than $l_{0}$, thus reducing the numerical overestimation of the fracture energy [37]. Time steps have been chosen sufficiently small to satisfy the conditional stability of the explicit scheme. Mesh size and time steps have been varied to ensure that converged results have been obtained. Besides, it has also been observed that energy conservation is ensured at a satisfying accuracy for small time steps as mentioned in [37].

Although the following results will be presented for a constant regularization length of $l_{0}=0.1 \mathrm{~mm}$, let us highlight that this value has been also varied. Diminishing this value resulted in a decrease of the damaged band widths as expected but crack patterns as well as crack velocities remained essentially the same. Indeed, as long as $l_{0} \ll L$ and the solution is localized in a damaged band, reducing $l_{0}$ will only produce a self-similar thinner damage profile in the direction normal to crack propagation whereas the crack will still propagate according to a similar energy release rate. 


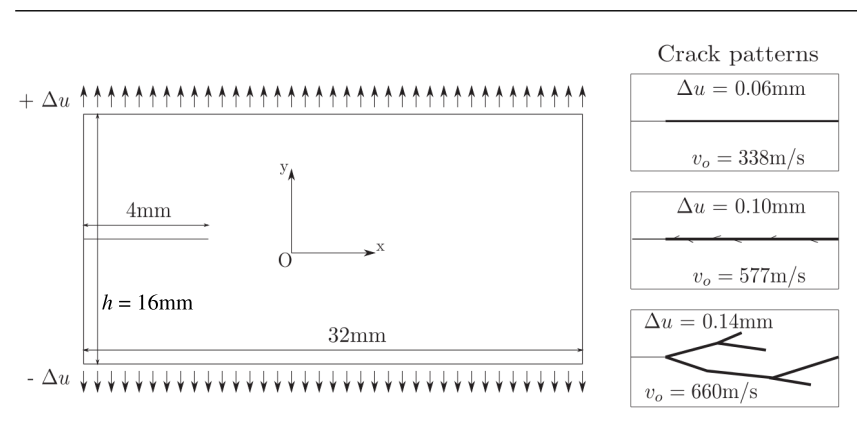

Fig. 3 Pre-strained PMMA plate problem (left) and crack patterns (right) sketched from experimental observations [72] for different imposed displacements (taken from [67]). For low loading levels, single crack propagation is observed. For higher values, microbranches appear during propagation. For even higher loadings, macroscopic branches are formed.

\section{Dynamic crack branching in homogeneous medium}

\subsection{Position of the problem}

We investigate the problem of dynamic crack propagation in a pre-strained PMMA rectangular plate geometry. This problem has been previously investigated experimentally in [72] (it is also quite close to the experiments of [60]) and from a numerical point of view using cohesive elements [73] and a non-local integral damage model [67]. Contrary to more traditional numerical benchmark problems in dynamic branching such as those investigated in $[7,8,29]$ where a constant stress echelon loading is applied, in this particular geometry, no stress waves are produced by the loading but only by crack propagation.

Indeed, the experimental setup of [72] consists, first, in moving the upper and lower surfaces of the plate in a quasi-static manner until reaching a desired displacement. While maintaining the upper and lower boundaries at this fixed value, a small sharp crack is created at the middle of the sample using a razor edge. The crack then accelerates until reaching a steady-state propagation regime. Another advantage of this problem is that the initially stored energy is well defined and can be related to the fracture energy dissipated by crack advance. This pre-strained strip configuration has been used in many experiments to measure the dependence of fracture energy on crack velocity. For all these reasons, we advocate this problem as a benchmark for assessing numerical models of dynamic brittle fracture.

The geometry and boundary conditions are represented in Figure 3 and are identical to those used in [73,67]: the plate is $32 \mathrm{~mm}$ wide and $16 \mathrm{~mm}$ high with a $4 \mathrm{~mm}$ pre-notch and modeled as a $2 \mathrm{D}$ plane-stress medium. It is pre-strained by applying uniform displacements $\pm \Delta U$ in the vertical direction on the bottom and top surfaces. First, a quasi-static computation is done to reach the pre-strained state, then an explicit dynamic computation is carried out to simulate the crack propagation.

PMMA is assumed to be isotropic linear elastic with $E=3.09 \mathrm{GPa}, \nu=0.35, \rho=1180 \mathrm{~kg} / \mathrm{m}^{3}$, the Rayleigh wave speed is $c_{R}=906 \mathrm{~m} / \mathrm{s}$. As regards the damage parameters, a value of $G_{c}=300 \mathrm{~J} / \mathrm{m}^{2}$ is retained, corresponding to quasi-static measurements and the regularization length is $l_{0}=0.1 \mathrm{~mm}$.

\subsection{A comment about the infinite strip configuration}

Let us mention that the previous problem is close to the infinite strip configuration, see [22] for instance. For this translation-invariant problem, a steady state propagation occurs with a fracture energy $\Gamma$ equal to the initially stored energy per unit length, given by $W=2 E(\Delta U)^{2} / h$ where $h$ is the plate height. This configuration seems thus appropriate to measure the possibly velocity-dependent fracture energy.

An approximate equation of motion for the infinite strip problem has been derived in [42] using a perturbation approach:

$\dot{v}=\frac{1-\Gamma(v) / W}{h / 2} c_{d}^{2}\left(1-v^{2} / c_{R}^{2}\right)^{2}$

As $W$ can be arbitrarily chosen, when $W>\max _{v} \Gamma(v)$ the crack is supposed to accelerate up to the Rayleigh wave speed but with an increasing effective mass so that acceleration becomes always more difficult when approaching $c_{R}$ [10]. The identification of $\Gamma(v)$ to $W$ can then only be valid for a true steady state. For high velocities, an even small value of acceleration can correspond to an important difference between $\Gamma(v)$ and $W$, leading to an overestimation of $\Gamma(v)$.

\subsection{Numerical computation of the macroscopic fracture energy}

The crack tip position has been recorded by considering the top-rightmost node of the finite element mesh for which the damage field is greater than 0.9 , its horizontal position is denoted by $a(t)$ with the origin taken at the prenotch tip $(a(0)=0)$. Hence, in the case of macroscopic branching, only the upper-branch tip is followed. Unless stated otherwise, the crack tip velocity $v$ 
corresponds to the horizontal component of the instantaneous velocity vector of the crack tip $\dot{a}$. This velocity is computed by fitting an affine function for a few simulation outputs around $a(t)$.

The damage dissipation rate $\Gamma$, which can be interpreted as a macroscopic fracture energy, has been computed as the derivative of dissipated energy per unit crack advance

$\Gamma=-\frac{d E_{e l}}{d a}-\frac{d E_{k i n}}{d a}=\frac{d E_{f r a c}}{d a}$

since the boundaries of the domain are fixed during crack propagation. $\Gamma$ has then been estimated by fitting an affine function for a few simulation outputs around $E_{\text {frac }}(a)$. Let us also mention that the apparent dynamic energy release rates can also be computed using the $G-\theta$ method (virtual domain extension) generalized to such damage-gradient models [36]. Despite slight differences, the values computed using such a method are consistent with those derived from (14).

\subsection{Dynamic crack branching results}

Simulations have been undertaken for different values of the imposed displacement $\Delta U$. Evolutions of crack velocities are reported in Figure 4 in which it can be observed that cracks accelerate up to a limiting velocity which is below the Rayleigh wave speed $c_{R}$, around $0.68 c_{R}$. The acceleration is stronger for higher loadings and it decreases when the crack speed approaches the final velocity, which is consistent with experimental observations and the infinite-strip equation of motion (13).

For small values of the loading $(\Delta U<0.04 \mathrm{~mm})$, no macroscopic branching has been observed during the simulation. For higher loadings, branching has been observed and it occurs sooner the higher the loading. phase-field distributions corresponding to different loading levels have been represented in Figure 5.

Let us observe that these simulations yield crack velocities that are higher than those observed experimentally for the same loading. As a consequence, macroscopic branching also occurs at lower loading levels than in the experiments. For instance, experiments with $\Delta U=$ $0.06 \mathrm{~mm}$ reported a steady state velocity of $0.37 c_{R}$ with a single crack propagation.

This discrepancy between simulations and experiments has always been observed in previous works using cohesive zone models [73] or non-local integral damage models [67]. Both works mentioned the lack of ratedependency of the constitutive model as the origin of this difference. Inclusion of a viscous dissipation energy, as initially introduced in [33] and proposed in [45] as a purely artificial regularization to improve numerical stability, would enable to introduce a rate-dependent effect reducing the value of the computed velocities. However, this aspect would be the purpose of another work and we will not attempt here to reproduce quantitatively the measured velocities but we will concentrate on other aspects of dynamic crack propagation such as the transition from single crack propagation to branching. Besides, we expect that the rate-dependency will have less influence for fast cracks reaching a steadystate for which the material response will be quasiinstantaneous contrary to slow or accelerating cracks. As the branching mechanism occurs at a high limiting velocity, rate-dependent effects may be less important in this context.

A striking result is that the damaged band widens as the crack propagates, which has also been previously reported in other phase-field simulations $[8,37]$ but also with other models such as a process-region cell model [32] or peridynamics [7]. This "widening" is mild for low loading levels but it is clearly visible for higher levels, especially before a branching event. For this reason, it is not easy to detect the exact origin of branching as it does not seem to be an abrupt phenomenon but rather a progressive one, exhibiting a continuous transition from an increasingly wider band to almost two crack tips propagating horizontally. At some point, when the two tips are sufficiently established, they start to screen and repel each other, leading to a true bifurcation and propagation of two isolated branches with a smaller damage band width. If the branching angle is measured as the angle made between the straight parts of the two isolated branches, a value close to $30^{\circ}$ is found, which is consistent with experiments and other simulations [7]. However, we have also observed that different values of the branching angle can be obtained by varying the domain geometry (it is smaller for plates with a smaller height $h)$.

The evolution of $\Gamma(t)$ as a function of the crack tip position $a(t)$ enables to better understand how the damaged band width increases with crack propagation. Indeed, denoting by $l(t)$ the total damaged surface (per unit length in the transverse direction), let us recall that

$$
E_{\text {frac }}(t)=G_{c} \int_{\Omega} \frac{3}{8 l_{0}}\left(d+l_{0}^{2} \underline{\nabla d} \cdot \underline{\nabla d}\right) d \Omega=G_{c} \cdot l(t)
$$




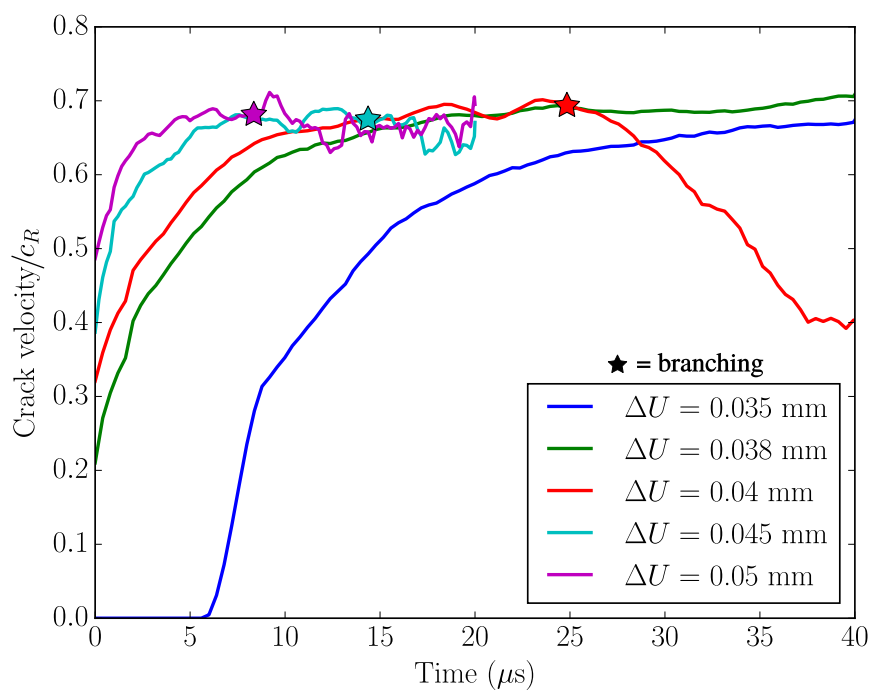

Fig. 4 Evolution of crack tip velocity for different loadings. For all loadings, the crack accelerates up to a limiting velocity around $0.68 c_{R}$. Branching events are indicated by the star-shaped symbol.

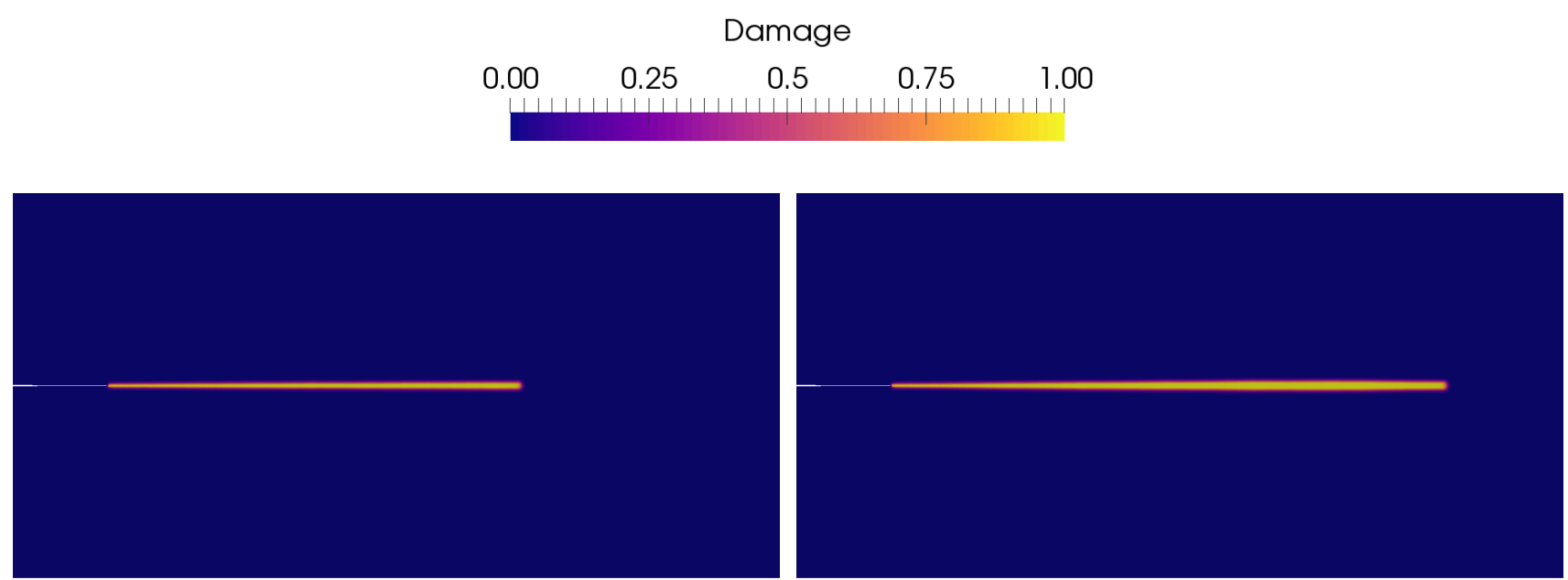

(a) $\Delta U=0.035 \mathrm{~mm}$ at $t=40 \mu \mathrm{s}$

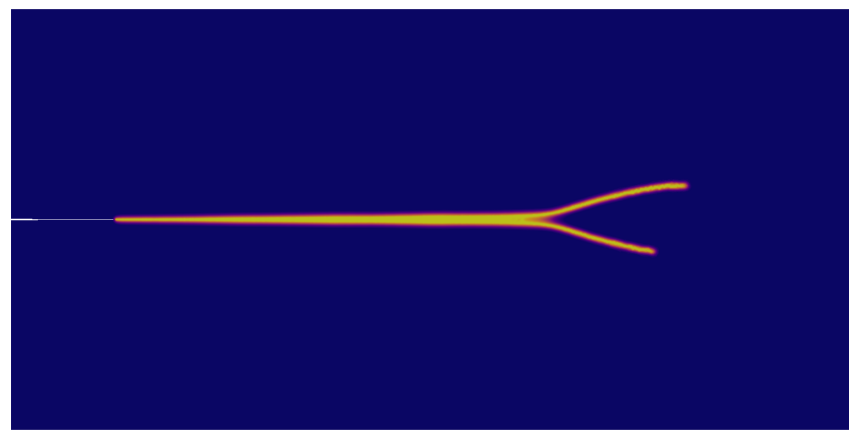

(c) $\Delta U=0.040 \mathrm{~mm}$ at $t=40 \mu \mathrm{s}$ (b) $\Delta U=0.038 \mathrm{~mm}$ at $t=40 \mu \mathrm{s}$

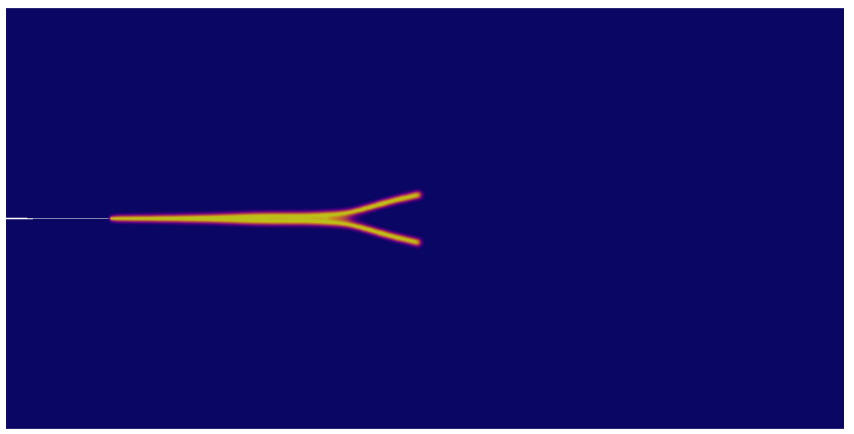

(d) $\Delta U=0.045 \mathrm{~mm}$ at $t=20 \mu \mathrm{s}$

Fig. 5 Phase-field distribution for different loadings. The damaged zone widens as the crack propagates and accelerates. For low loading levels, a single crack propagation is observed and macroscopic branching is observed for higher loadings. It occurs earlier in the crack propagation for higher loadings. The branching angle is around $30^{\circ}$. 


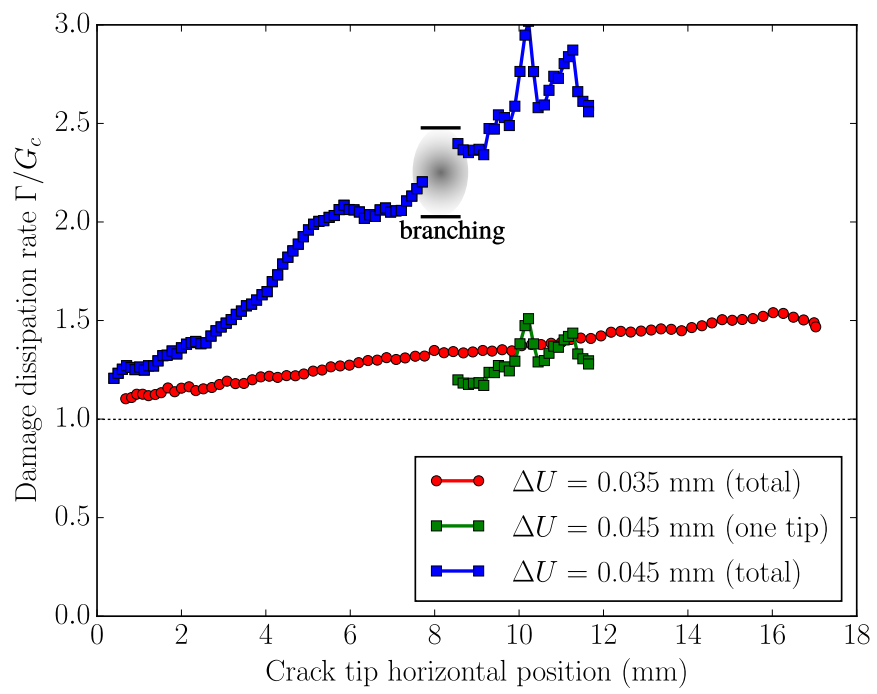

Fig. 6 The normalized damage dissipation rate $\Gamma / G_{c}$ increases during crack propagation. For the low loading level $(\Delta U=0.035 \mathrm{~mm})$, the evolution is regular with $G_{c} \leq \Gamma \leq 1.5 G_{c}$ and no branching is observed. This increase leads to an effective thickening of the crack width. For a higher loading $(\Delta U=0.045 \mathrm{~mm})$, branching is observed slightly after $\Gamma \geq 2 G_{c}$, corresponding to two crack tips. After branching, the dissipation associated with a single crack tip $(\Gamma / 2)$ is close to its initial value, slightly above $G_{c}$.

since $G_{c}$ is uniform in the whole sample. Therefore, $\Gamma(t)=G_{c} \frac{d l}{d a}$, so that the normalized damage dissipation rate $\Gamma / G_{c}$ is equal to the increase in total fracture surface $l(t)$ per apparent crack surface increase (if one interprets $a(t)$ as an apparent crack surface). This quantity has been represented in Figure 6 for two different loading values: $\Delta U=0.035 \mathrm{~mm}$ for which no macroscopic branching takes place and $\Delta U=0.045$ $\mathrm{mm}$ for which macroscopic branching is observed. After a first phase of initiation, the crack advances with values of $\Gamma$ slightly above $G_{c}$. The damage dissipation rate then increases during crack advance with an almost constant rate depending on the loading level. As mentioned before, this increase is directly related to the increase of total over apparent fracture surface due to the damaged band widening. It is to be noted that, for the duration of the simulation with $\Delta U=0.035 \mathrm{~mm}, \Gamma$ increased from $G_{c}$ to $1.5 G_{c}$ and no branching has been observed. For the higher loading $(\Delta U=0.045 \mathrm{~mm})$, branching has been observed slightly after $\Gamma$ exceeded $2 G_{c}$, a value corresponding to two crack tips, although detection of the exact instant of branching is difficult (this is symbolized by the gray zone in Fig. 6, the corresponding values of $\Gamma$ have not been represented during the transition from single to branched crack). After branching, the total damage dissipation rate continued to increase and when looking only at the contribution of a single branch (given by $\Gamma / 2$ due to the symmetry of the branched pattern, see Fig. $5(\mathrm{~d})$ ), values close to the initial ones (slightly above $G_{c}$ ) are obtained. This last observation is related to the fact that the damage band width of the branched parts are smaller than the main crack just before branching. Finally, the increase of $\Gamma / 2$ after branching suggests that a new branching event can occur if this quantity again reaches a critical value close to $2 G_{c}$.

We are not aware of previous works reporting such numerical results pointing towards an energetic criterion for the occurrence of branching. In particular, our results indicate that branching can be triggered without stress waves arriving at the crack tip as already indicated in [7]. However, incoming waves at the crack tip can lead to a sudden increase of the dynamic energy release rate, which will exceed its critical value and form branches. This seems to be the case for the more traditional stress echelon problem $[7,8,30]$.

When inspecting damage profiles in a direction perpendicular to the main crack propagation for $\Delta U=$ $0.04 \mathrm{~mm}$ (see Fig. 7), it can clearly be observed that, initially, the damaged zone is close to the one-dimensional analytic damage profile $d(y)=\left(1-|y| /\left(2 l_{0}\right)\right)^{2}(\mathrm{~A})$, thus explaining why $\Gamma \approx G_{c}$ at the early stage of propagation. A fully damaged zone $(d=1)$ with increasing width then appears during crack advance (B and $\mathrm{C}$ ). It is to be noted that the shape of the transition zone from $d=0$ to $d=1$ remains similar, at all stages, to the theoretical 1D damage profile. The associated increase in $\Gamma$ before branching is, thus, essentially due to this wide zone with $d=1$. At the onset of branching, one can observe that the central region exhibits a 


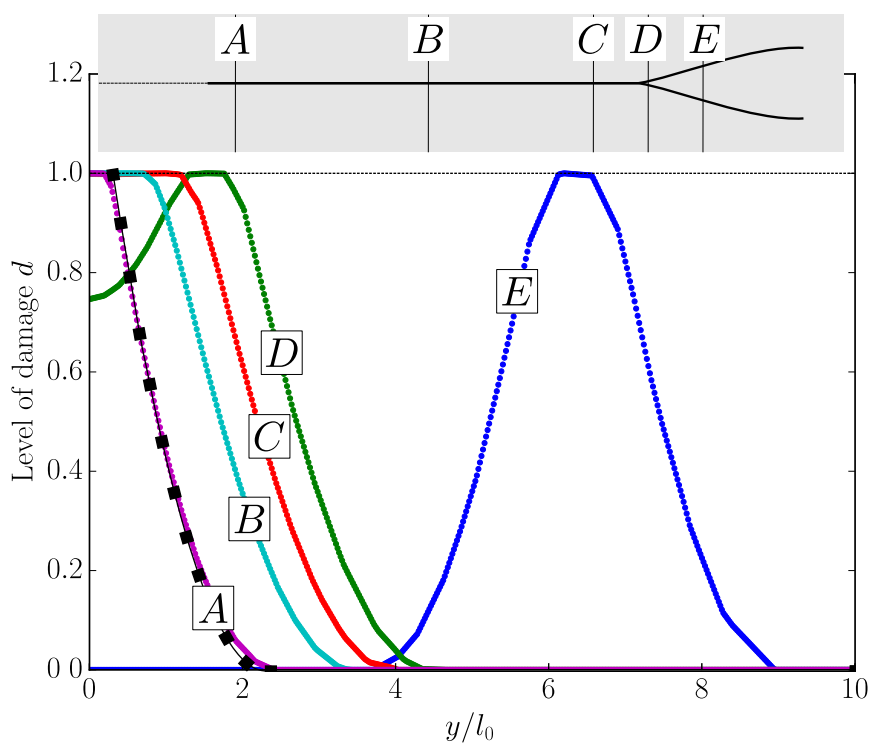

Fig. 7 Symmetric vertical damage profiles for $y \geq 0$ at different positions from the left part of the domain $(\Delta U=0.04$ mm): $A(x=5 \mathrm{~mm}), B(x=12 \mathrm{~mm}), C(x=18 \mathrm{~mm}), \bar{D}(x=20 \mathrm{~mm}), E(x=22 \mathrm{~mm})$. The profiles clearly indicate the existence of a fully damaged zone $(d=1)$ with increasing width before splitting into two distinct cracks. The black square line corresponds to the $1 \mathrm{D}$ parabolic profile.

damage level lower than 1 , the maxima of the damage profile occurring at two symmetric positions away from the central path, as if two single crack tips were propagating side-by-side (D). With a further reduction of the central zone damage level, the two tips are well isolated and start to mutually screen each other, leading to a symmetric deviation of the two branches from the horizontal direction. After branching (E), the damage profile of a single branch is similar to the initial profile with a small fully damaged band.

Let us highlight that, for all simulations, the crack propagates from left to right with an already fully established damage band width, meaning that this wide band does not appear after the crack tip has further propagated. Hence, these two modes of propagation (crack tip advance and damage band widening) occur simultaneously and, once the crack tip further advances, no further evolution of the damage band is observed in the crack tail.

Let us briefly discuss here the effect of the mesh size on the different damage profiles and the computed value of $\Gamma$. In these simulations, a mesh size of $h=$ $0.02 \mathrm{~mm}$ has been used near the middle plane of crack propagation corresponding to 5 elements over a distance of $l_{0}$. In $[12,37]$, it has been mentioned that the quasistatic damage profile can be wider than the theoretical one by approximately $\delta_{h} \approx 2 h$ (see Fig. 8-top) leading to the following overesimated fracture energy:

$\Gamma_{e s t}^{q s}=G_{c}\left(1+\frac{3(2 h)}{8 l_{0}}\right)=G_{c}+\Gamma^{m e s h}$

In our case, the quasi-static fracture energy would be over-estimated by $15 \%$. In practice, a smaller overestimation (around 10\%) is observed, see for instance the measured value of $\Gamma \approx 1.1 G_{c}$ in Fig. 6 for $\Delta U=0.035$ $\mathrm{mm}$ at the beginning of crack propagation. In the dynamic case, we observe a similar behavior i.e. the two sharp transitions from the fully damaged zone to the parabolic profile can be over-estimated by a distance $h$ (see Fig. 8-bottom). As a result, if we consider a damage profile consisting of a fully damaged zone of width $2 s$ due to dynamic effects, two mesh-related overestimations of size $\delta_{h} \approx h$ and the two parabolic profiles, then the effective dynamic fracture energy can be estimated as:

$\Gamma_{\text {est }}^{d y n}=G_{c}(\underbrace{1+\frac{6 s}{8 l_{0}}}_{\text {dynamic }}+\underbrace{\frac{6 h}{8 l_{0}}}_{\text {mesh size }})=\Gamma^{d y n}+\Gamma^{m e s h}$

where $\Gamma^{\text {mesh }} \approx 0.15 G_{c}$. The previous expression of $\Gamma^{d y n}$ holds only in the case of a single-crack propagation (profiles A to $\mathrm{C}$ ). In the transition regime (profile D), the decrease of damage at $y=0$ should be taken into account. 

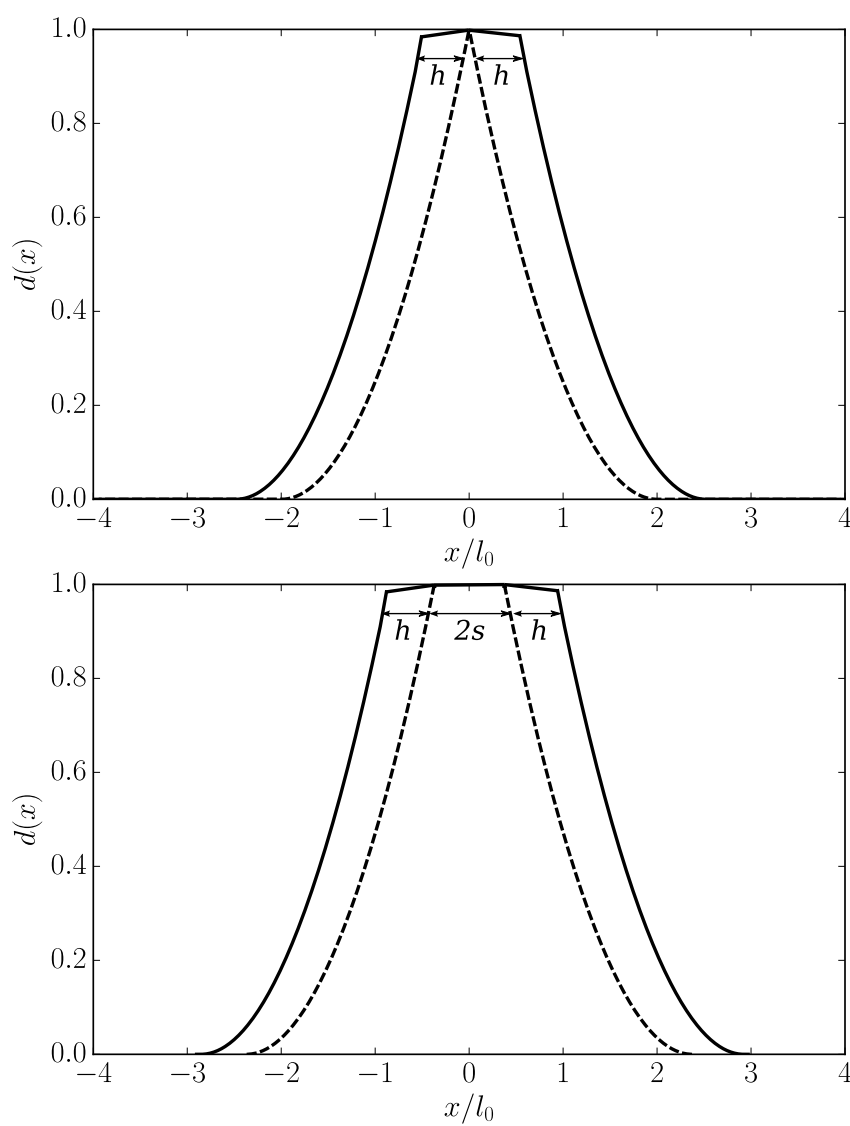

Fig. 8 Potential effect of mesh size (solid line) on the damage profiles (dashed lines without mesh-size effect): quasi-static case (top) and dynamic case (bottom).

Except in one case $(\Delta U=0.04 \mathrm{~mm})$, no evident decrease of the crack velocity has been observed after branching. Although such results are not reported here, simulations in different configurations (loading, boundary conditions) exhibited branching at much lower velocities than those reported here (around $0.2 c_{R}$ ). These results suggest that the crack velocity is not a determining parameter for crack branching. However, a critical value of the energy release rate (close to $2 G_{c}$ ) seems to be a necessary condition for branching for this phasefield model. After branching, the damage rate drops to a value close to $G_{c}$, which is associated with a smaller damaged zone, until the process eventually repeats itself (multiple branching events have indeed been obtained for higher loading values). This mechanism is very similar to the one suggested in [54].

In view of the present results, we believe that an energetic criterion is a pertinent way to describe the branching process and would, therefore, require further investigations, either from numerical simulations or experiments. In particular, it would be very interesting to design experiments with patches of different materials with a known fracture energy: one can think of a situation in which a single crack propagates in a tougher material and branches when arriving in a weaker one.

\subsection{Velocity-toughening results}

For all loadings, the evolution of the damage dissipation rate $\Gamma$ as a function of the instantaneous crack velocity $v$ has been recorded during the single crack propagation i.e. slightly after the initiation phase and before the occurrence of macroscopic branching. Both quantities have been represented in Figure 9. A well-defined master-curve is obtained for all loading levels showing that $\Gamma$ is slightly greater than the quasi-static fracture energy $\Gamma(0)=G_{c}$ at low velocities whereas a strong increase of $\Gamma$ can be observed when approaching a limit speed around $0.7 c_{R}$. Remarkably, this value is close to the limiting velocity of $0.75 c_{R}$ identified experimentally in [72] for the same geometry and loading conditions.

The L-shape aspect of the curve is associated with a competition between two behaviours already identified in dynamic crack propagation experiments: at low velocities, a single crack propagating according to Griffith theory whereas, at high velocities, a constant velocity regime with important increase of additional dissipation mechanisms at the crack front.

This observation can be linked to the experimentally observed transition between a mirror-like propagation and a mist then hackle propagation characterized by surface roughening and microbranching. Although the present $2 \mathrm{D}$ model is not able to resolve the small length scales associated with the onset of microbranches (and obviously its 3D nature) or surface roughening, it is possible for the damage field evolution to "choose" (as a by-product of energy minimization) between dissipating more energy by advancing the crack tip at a higher velocity or by increasing the width of the damaged zone by propagating a damage front on a small distance perpendicularly to the main crack propagation (this last possibility could be associated with some kind of crack tip blunting).

The degree of generality of Figure 9 has been addressed by changing the material parameters to those close to Homalite-100 with $E=4.55 \mathrm{GPa}, \nu=0.35$, $\rho=1230 \mathrm{~kg} / \mathrm{m}^{3}$ and $G_{c}=38.5 \mathrm{~J} / \mathrm{m}^{2}$ and to soda-lime glass with $E=72 \mathrm{GPa}, \nu=0.22, \rho=2440 \mathrm{~kg} / \mathrm{m}^{3}$ and $G_{c}=3.8 \mathrm{~J} / \mathrm{m}^{2}$ while keeping the same geometry. In these cases, the Rayleigh wave speed is $c_{R}=1093 \mathrm{~m} / \mathrm{s}$ for Homalite and $c_{R}=3102 \mathrm{~m} / \mathrm{s}$ for glass. We also varied the PMMA plate geometry by considering a square 


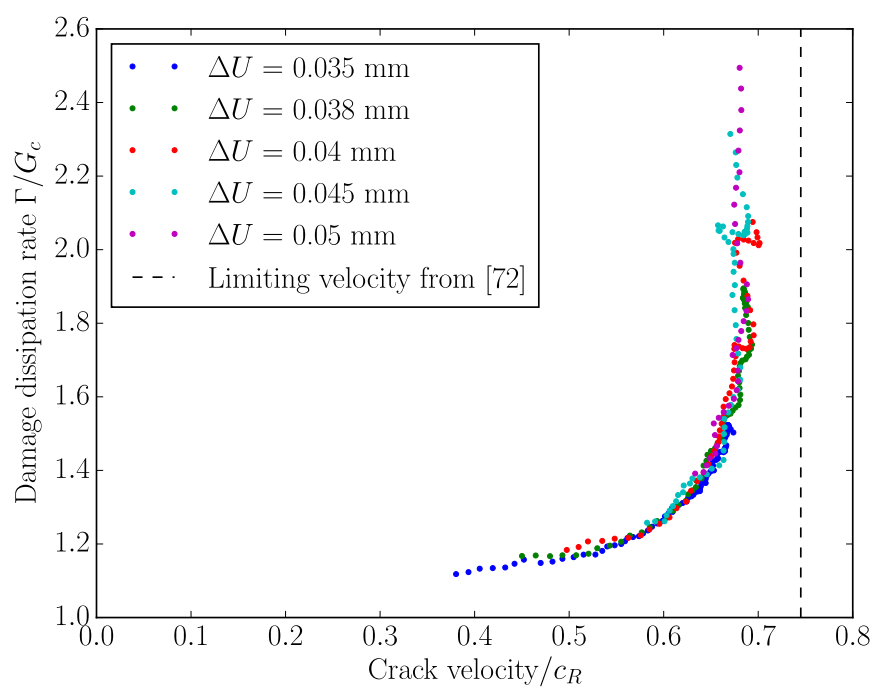

Fig. 9 The damage dissipation rate $\Gamma$ is a well-defined increasing function of the crack speed for all initial loadings. The points correspond to instantaneous values of $v$ and $\Gamma$ during the single crack propagation phase i.e. after initiation phase and before branching for high loadings. The dotted line corresponds to the experimentally observed limiting velocity in [72].

geometry of $32 \mathrm{~mm} \times 32 \mathrm{~mm}$ and a slender band of 32 $\mathrm{mm} \times 8 \mathrm{~mm}$ with the same pre-notch of $4 \mathrm{~mm}$.

The $\Gamma(v)$ relation is represented in Figure 10 for these different cases along with the collective results of Figure 9. It can be observed that the same increasing trend is obtained for each case. Interestingly, the non-dimensional results corresponding to Homalite-100 and glass superimpose quite well to those obtained with PMMA for the same geometry. On the contrary, it seems that the $\Gamma(v)$ relation is more dependent on the geometry, at least at high velocities.

The origin of this dependence on geometry is not yet clear, it may certainly be related to a different interaction of the crack with stress waves reflected at the boundary of the domain. In any case, it is a signature of a difference in the conversion process of kinetic and elastic energies into fracture energy. It is interesting that such a process seems independent of material properties when represented in terms of non-dimensional variables while elastic moduli, fracture energies and sound wave speeds differ by several factors.

Interestingly, without including any rate-dependency in the model, our results are able to reproduce a velocitytoughening mechanism at the macroscopic scale. Although this increase of fracture surface seems to be limited to a factor 2.5 whereas in [60] a factor 6 has been observed due to the creation of microbranches, our simulations show that a limiting velocity consistent with experimental observations can be achieved. For instance, this was not the case with rate-independent cohesive models [73] in which cracks could accelerate up to the Rayleigh wave speed for this particular configuration.

\section{Crack propagation in a heterogeneous medium}

Brittle fracture mechanics in heterogeneous materials is now receiving increasing attention and more specifically in the dynamic case for which numerous questions are still unanswered. The role of heterogeneities in dynamic fracture processes is studied across various length scales, ranging from kilometres for earthquakes [20,35], to millimetres for composite plates [71] or heterogeneous thin films [64] or even to nanometres in metallic glasses [48]. Heterogeneities can have a complex influence on a crack propagation by nucleating daughter cracks or exhibit a transition from weak to strong pinning regime [50]. In particular, all these various dissipative mechanisms in presence of heterogeneities make it difficult to predict the effective toughness of a heterogeneous material.

In this section, we revisit the limiting speed, branching and velocity-toughening mechanism in presence of heterogeneities. As mentioned previously, experiments have also reported that crack velocities near $c_{R}$ can be obtained when constraining the crack path to a weaker interface or by drilling an array of holes ahead of the crack [66]. We first aim at reproducing numerically these observations. The velocity-toughening mechanism is then reinterpreted for a crack propagating along such an interface and subjected to unsuccessful branching at- 


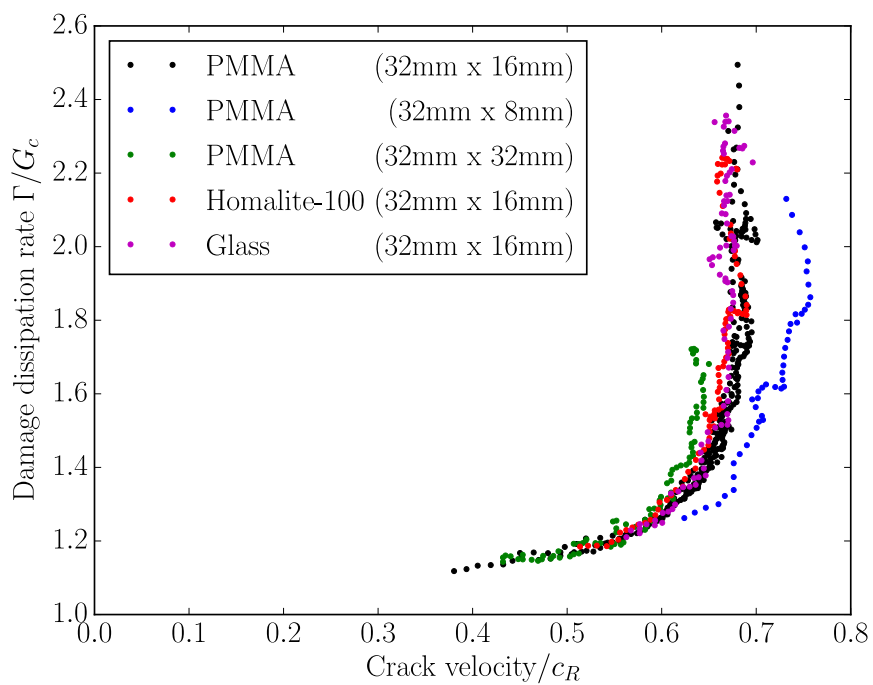

Fig. 10 The normalized damage dissipation rate $\Gamma / G_{c}$ as a function of the normalized crack velocity $v / c_{R}$ does not seem to depend on material properties whereas it is dependent on geometry.

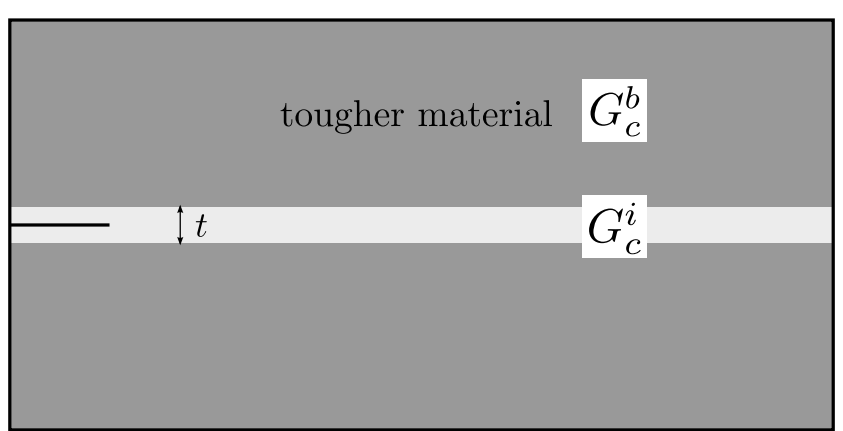

Fig. 11 Constrained propagation along a weak interface: the same problem as in Section 3 is considered, except that the crack is forced to propagate along the weak interface of fracture energy $G_{c}^{i}$, the surrounding material has the same elastic properties but is much tougher so that macroscopic branching is prevented.

tempts. Finally, we also investigate how a distant heterogeneity can influence the crack propagation path.

4.1 Constrained crack propagation along a weak interface

First, a band of material of width $t=2 l_{0}=0.2 \mathrm{~mm}$ and fracture energy $G_{c}^{i}=300 \mathrm{~J} / \mathrm{m}^{2}$ surrounded by a tougher material $\left(G_{c}^{b}=100 G_{c}^{i}\right)$ is considered (see Fig. 11). Elastic properties are still homogeneous and correspond to those of Section 3 so that the initial prestressed state is the same as before.

In this configuration, the crack never branched (Fig. 12). Contrary to Fig. 7, the phase field is almost fully saturated to $d=1$ with a steep transition to $d=0$ at the interface between both materials. For a given

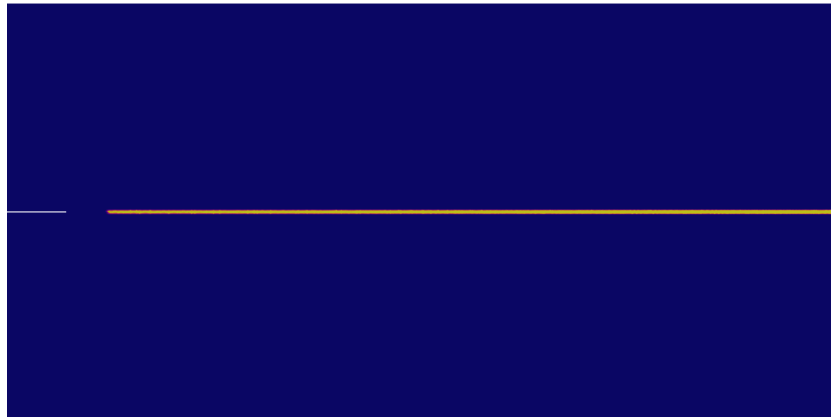

Fig. 12 Crack propagation in a constrained path configuration with $\Delta U=0.05 \mathrm{~mm}$. Branching is completely suppressed, dissipation takes place only inside the middle interface and the crack accelerates up to the Rayleigh wave speed.

Table 1 Crack velocities for the weak interface configuration. Velocities up to $0.98 c_{R}$ have been attained.

\begin{tabular}{ccc}
\hline$\Delta U(\mathrm{~mm})$ & Stored energy $\left(\mathrm{J} / \mathrm{m}^{2}\right)$ & Crack velocity $\left(c_{R}\right)$ \\
\hline 0.04 & 618 & 0.81 \\
0.05 & 966 & 0.87 \\
0.10 & 3,863 & 0.94 \\
0.15 & 8,691 & 0.98 \\
\hline
\end{tabular}

loading, the crack is accelerating to higher velocities than for unconstrained configurations. Final velocities for different initial loadings are reported in Table 1. In particular, it has been possible to obtain velocities up to $0.98 c_{R}$ for the highest loading. It is to be noted that for $\Delta U>0.15 \mathrm{~mm}$, the whole interface starts to damage at the same time due to the initial stress state exceeding the elastic limit of the damage model. 


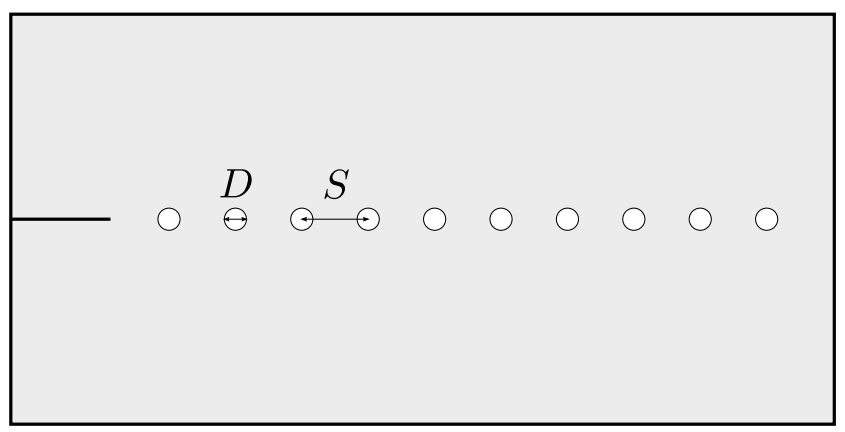

Fig. 13 Constrained crack propagation in a perforated material: here the same homogeneous material as in Section 3 is considered except that an array of holes has been drilled in front of the crack path. This results in an apparently weaker interface so that propagation is also favoured along this plane.

\subsection{Crack propagation through a perforated material}

We now go back to the case of the homogeneous material of Section 3 except that an array of holes of diameter $D$, spaced by a distance $S$, is considered on the mid-plane of the sample ahead of the crack tip (Fig. 13). We considered 30 holes with $D=0.4 \mathrm{~mm}$ and $S=0.9 \mathrm{~mm}$ so that the holes are sufficiently small for inducing only a negligible change of the total initially stored elastic energy and sufficiently weaken the middle interface. Although this configuration induces stress concentrations at the hole boundaries, the stress levels remain sufficiently low so that no damage occurs between holes prior to the crack tip arrival.

Crack propagation is driven by a succession of the following events (Fig. 14): when arriving near a hole, the crack tip is first attracted to the boundary; after a short time without any further evolution of the phase field, a new crack nucleates on the middle plane at the opposite point of the hole boundary and starts to propagate towards the next hole.

As for the homogeneous case, progressive widening of the damage band can be observed with increasing crack advance (events A to $\mathrm{C}$ ); for sufficiently available energy at the crack tip, this can translate into an attempt at forming two branched cracks at the nucleation point (events $\mathrm{C}$ to $\mathrm{E}$ ). The attraction exerted by the next hole can then stop the branching process and reform one single crack from the two branches. As the crack further propagates, the length of the side branches increases. Similarly to the weakened interface, higher crack speeds can be obtained in this configuration. For instance, an initial loading of $\Delta U=0.05 \mathrm{~mm}$ leads to averaged velocities up to $0.9 c_{R}$ (Fig. $15(\mathrm{a})$ ).

It is interesting to note that this particular simulation shares some qualitative similarities with the micro- branching phenomenon when considering the role of nucleation, growth and coalescence of microcracks ahead of the main crack path $[57,58,16]$.

This process can be further understood when looking at the evolution of the damage dissipation rate during crack propagation (Fig. 15(b)). The five representative events are also reported and correspond approximately to the moment when the crack tip is located halfway between two holes.

At first, the apparent damage dissipation rate is less than the medium fracture energy due to the weakening presence of the holes. The apparent porosity along the main crack path is $\phi=D / S \approx 0.44$, a simple crude estimate of the weakened interface fracture energy using a rule of "mixture" would then be $G_{c, \text { weak }}=(1-\phi) G_{c}=$ $0.56 G_{c}$. This estimate is roughly consistent with the measured value of $\Gamma$ at the beginning of the propagation (after the initiation phase) in Fig. 15(b), which is around $0.7 G_{c}$ (event $\mathrm{A}$ ). It is also interesting to note that the increase of $\Gamma$ is relatively regular in the early stages of propagation, corresponding to a straight propagation of the crack through the first holes (up to event B). However, when the crack further propagates, bursts of increasing amplitude can be observed in the evolution of $\Gamma$, which can be directly associated with unsuccessful attempts at forming macrobranches (events $\mathrm{C}$ to $\mathrm{E}$ ).

From a macroscopic point of view, the evolution of $\Gamma$ during crack propagation can be related to the observations of Fig. 6 in the homogeneous case. One important difference is that the increase of $\Gamma$ is more important since it goes approximately from $0.7 G_{c}$ to $2.3 G_{c}$. Referring to Fig. 14 from a macroscopic point of view, this stronger relative increase in $\Gamma$ can also be associated with a widening of a macroscopic damage band. This widening is here essentially caused by unsuccessful branching attempts, with increasingly longer side branches as the crack propagates. It may seem surprising that a weakened interface would lead to a stronger increase in $\Gamma$. However, we might argue that, although the initial crack propagation phase can be associated with an effective fracture energy $G_{c, \text { weak }}$, the attractive presence of the holes makes it more difficult for a crack to branch. Assuming the existence of an energetic branching criterion like $\Gamma \geq G_{c, \text { branch }}^{\text {heter }}$, we may suppose that $G_{c, \text { branch }}^{\text {het }}$ is essentially the same as for the homogeneous case (because branching occurs inside the bulk between two holes) or is even higher since the holes attraction tend to delay branching. Thus, in this heterogeneous case, $\Gamma$ would increase from $G_{c, \text { weak }}<G_{c}$ to $G_{c, \text { branch }}^{\text {heter }} \geq G_{c, \text { branch }}^{\text {homog }}$. The presence of heterogeneities along the crack path has, thus, an important influence 


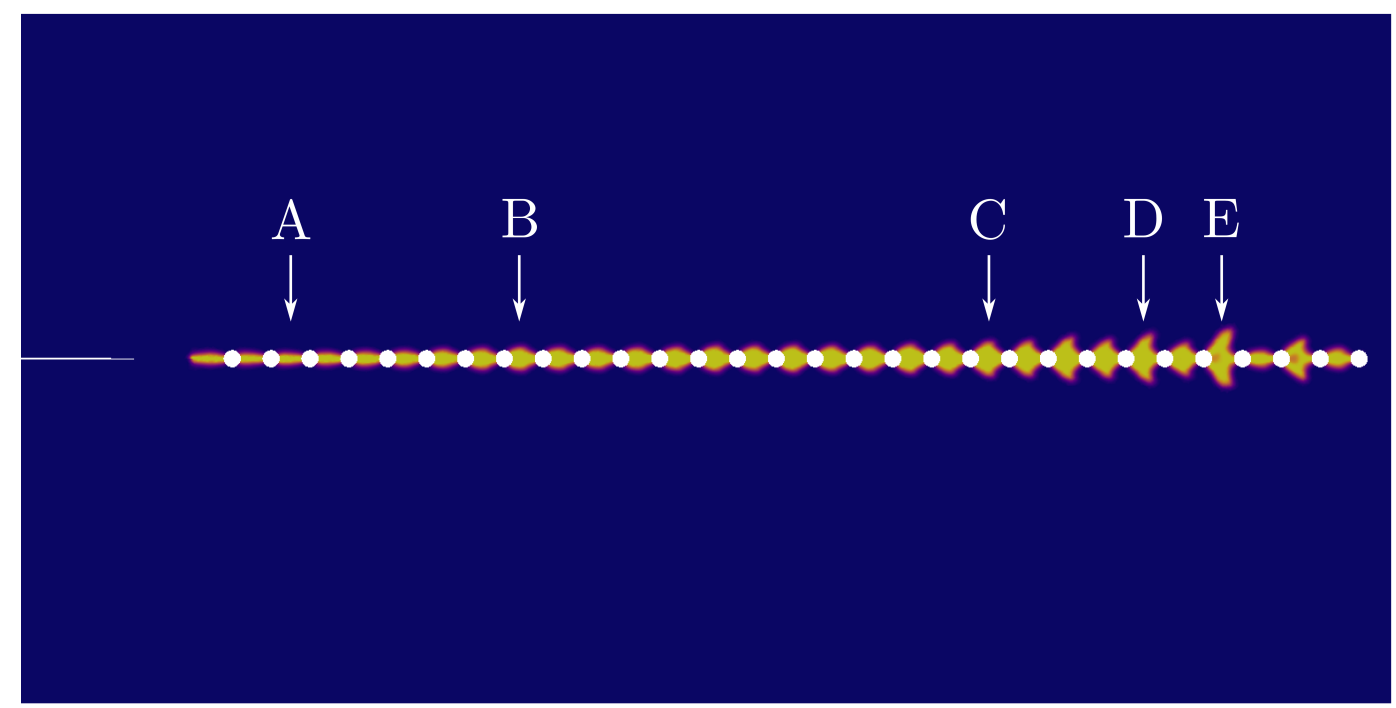

Fig. 14 Crack propagation in a perforated material with $\Delta U=0.05 \mathrm{~mm}$ (the same colormap as in Fig. 5 is used). Macrobranching is temporarily suppressed by crack tip attraction to the holes. Small attempts of branching can be observed at the end (events D and E). See also Movie 1 in supplementary material.

on the velocity-toughening mechanism.

When looking at the evolution of the different forms of energy (elastic, kinetic and dissipated through damage propagation), it can be observed that less energy is dissipated into fracture surface (Fig. 16 for $\Delta U=0.05$ $\mathrm{mm}$ ) when the crack path is constrained either in a weak interface or an array of holes than in the homogeneous case where macroscopic branching is possible. The excess amount is then mostly transferred into kinetic energy which can be linked to higher crack velocities.

As mentioned before, when approaching a hole, the crack tip is attracted to its boundary. This results in an increase of its instantaneous velocity before being momentarily stopped by the hole. In order to have a closer look at the interaction between the crack and a hole, we considered a simulation with 10 holes $(D=0.4$ $\mathrm{mm}$ ) separated by a distance $S=2.55 \mathrm{~mm}$, the local increase of the crack tip velocity when approaching each hole can clearly be observed in Fig. 17. Associated with this increase of crack tip velocities, strong bursts of kinetic energy can also be observed just before the crack encounters a hole (inset of Fig. 17).

Note also that, for this configuration, the time interval separating the moment the crack stops at the hole boundary and nucleates again at the opposite point is measured to be $\Delta t=0.694 \mu \mathrm{s}$, which corresponds exactly to the time for a Rayleigh wave to travel half of the hole circumference: $\Delta t=\pi D /\left(2 c_{R}\right)$.
4.3 Crack propagation in presence of distant heterogeneities

We investigate the influence of distant heterogeneities on the crack propagation path. The same geometry for the PMMA plate is considered here with different hole configurations. We present only preliminary results in the case of holes, although other kind of heterogeneities such as stiffer inclusions have also been considered. Investigating the mechanisms driving the interaction between a crack and various heterogeneities will require a more thorough study, which will be the purpose of another work.

First, we considered one single hole of diameter $D=$ $0.4 \mathrm{~mm}$, the center of which is situated $0.6 \mathrm{~mm}$ away from the middle plane of the sample and either $1 \mathrm{~mm}$ or $6 \mathrm{~mm}$ away from the pre-notch tip $(\Delta U=0.04$ $\mathrm{mm}$ ). Recalling that cracks accelerate progressively until reaching a limiting speed (Fig. 4), the crack will pass near the hole situated $1 \mathrm{~mm}$ from the pre-notch tip with a smaller velocity than when passing near the one situated $6 \mathrm{~mm}$ away from the notch. The comparison of the different crack paths between the two configurations is reported in Figure 18. It can be observed that for the closest hole, the crack interacts with the hole at a slower velocity and is only slightly deviated towards the hole but then continues its straight propagation. In the other case the crack arrives near the hole with a higher velocity, a microbranch appears and is attracted then stopped by the hole. The main part of the crack then continues its propagation with a smaller damage band 


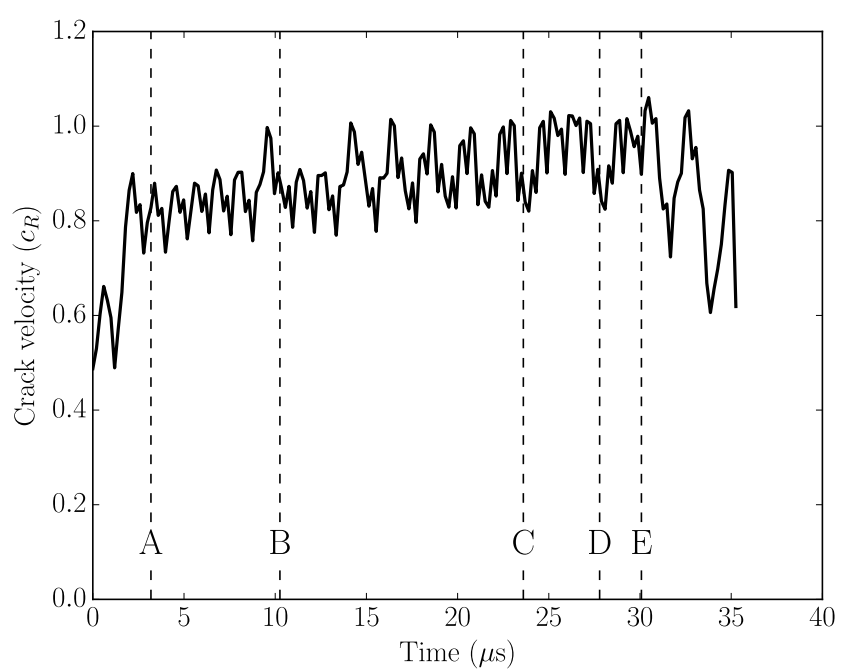

(a)

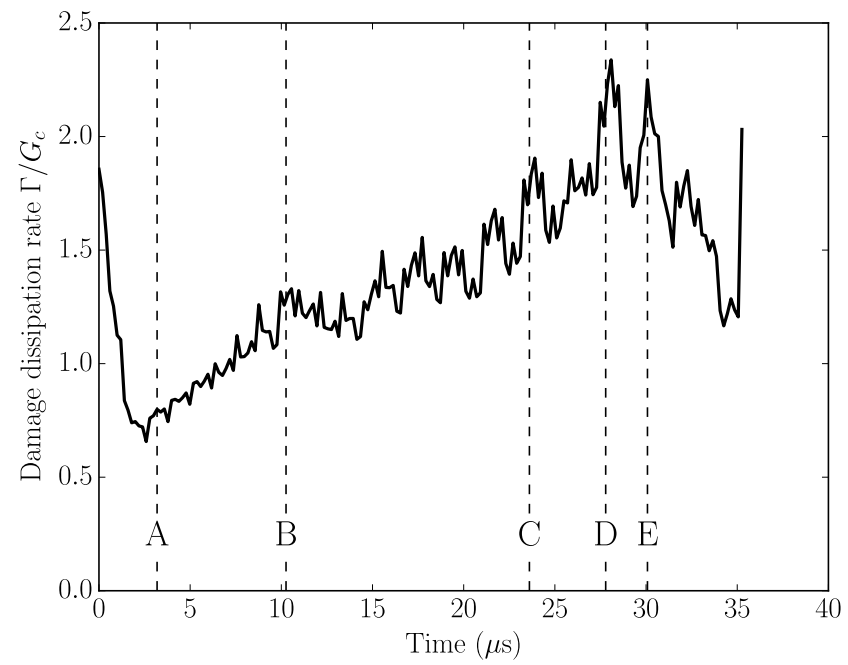

(b)

Fig. 15 Crack velocity (a) and damage dissipation rate (b) evolution during propagation through an array of holes $(\Delta U=0.05 \mathrm{~mm})$. Events A to $\mathrm{E}$ of Fig. 14 are reported using dashed lines. The damage dissipation rate increases with crack propagation and large bursts can be associated with unsuccessful branching attempts (events D and E). Velocities close to the Rayleigh wave speed are obtained.

width after the microbranching event. In both cases, a decrease of the instantaneous crack tip velocity can be observed when interacting with the hole. The crack accelerates again after passing the hole. Let us mention that the opposite is observed in the case of a stiffer inclusion, the crack accelerates when passing near the inclusion and then decelerates.

These results are consistent with known results regarding attraction by holes and deflection by stiff inclusions. However, one may have expected that a fast crack will have less time to interact with a distant heterogeneity than a slower one, contrary to what is observed.

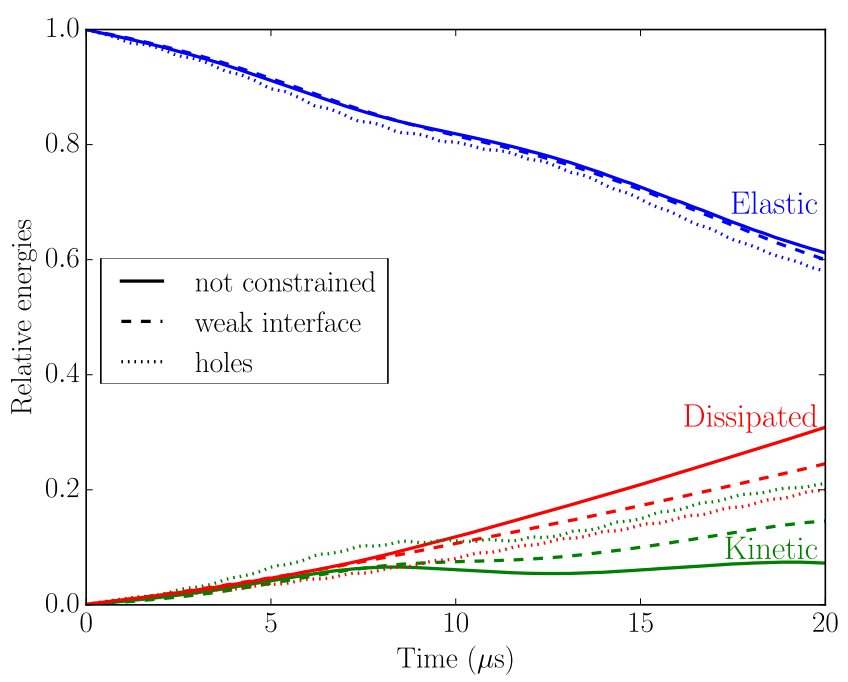

Fig. 16 Evolution of the different contributions to the total energy for $\Delta U=0.05 \mathrm{~mm}$ (normalized by the initially stored elastic energy): elastic (blue), kinetic (green) and dissipated (red) for the unconstrained case (solid) and weak interface (dashed) and array of holes layout (dotted). Dissipated energy is less important in the case of a constrained crack path, the difference being mostly compensated by an increase in kinetic energy and only slightly by a drop of elastic energy.

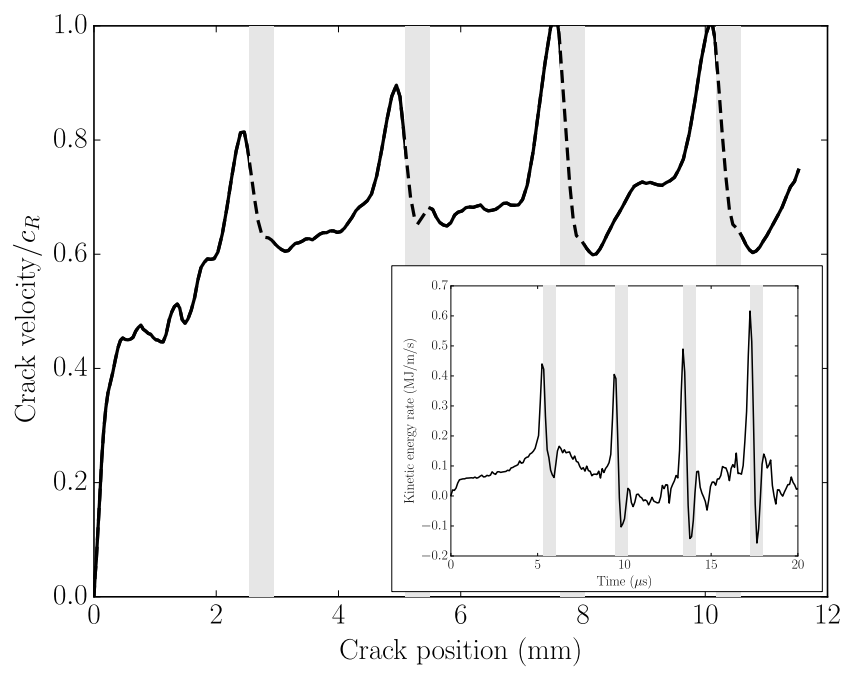

Fig. 17 Increase of the instantaneous crack tip velocity when approaching holes (gray bands) for $\Delta U=0.05 \mathrm{~mm}$. The dashed parts are due to the smoothing of the velocity when the crack is stopped by a hole. Inset: Rate of kinetic energy change as a function of time. Strong bursts of kinetic energy at a macroscopic level are associated with the crack accelerations when approaching a hole.

On the other hand, the velocity-toughening mechanism suggests that a faster crack seeks additional dissipation mechanisms than just a straight propagation at higher velocities. As the damage band widens, the distance at which it can interact with a defect may also increase. However, we do not have yet a clear explanation of these 
observations but we believe that it is a striking example of interesting interactions between fast cracks and heterogeneities, which therefore calls for further numerical or experimental investigations.

Finally, we have considered a configuration of 15 small holes of diameter $D=0.4 \mathrm{~mm}$, the centers of which are situated either $0.5 \mathrm{~mm}$ or $0.6 \mathrm{~mm}$ away from the middle plane of the sample. The spacing between two consecutive holes is $S=1.95 \mathrm{~mm}$. Two different loadings of $\Delta U=0.04$ and $0.05 \mathrm{~mm}$ have been considered.

The results for these configurations are reported in Figure 19. It can first be observed that the value of the considered offset has an important influence on the crack path patterns although the two geometric configurations are quite similar. In the first case of a $0.5 \mathrm{~mm}$ offset (Fig. 19(a) and (b)), the crack localizes in the weak plane composed by the holes when arriving near the first one. The propagation, is then similar to the one already described in the previous subsection with attempts at branching and attraction of the different branches by the next holes.

On the contrary, in the second configuration with a $0.6 \mathrm{~mm}$ offset and $\Delta U=0.04 \mathrm{~mm}$ (Fig. 19(c)), the crack is slightly attracted by the presence of the first hole but then continues its straight propagation while small microbranches emerge from the main path towards the next holes. For $\Delta U=0.05 \mathrm{~mm}$ (Fig. 19(d)), this behavior is more pronounced and the emergence of microbranches tend to deflect the main crack away from the holed plane. Additional microbranches can emerge and are attracted by the holes even when the main crack is quite far from the holed plane. As a result, the toughest plate corresponds to the second case with a $0.6 \mathrm{~mm}$ offset since the crack does not propagate through the weakened plane.

It is therefore quite surprising that, despite two configurations being almost similar, such different crack patterns are observed. Either the main crack is attracted by the hole and propagate along a weaker plane or it is repulsed by it and only microbranches are attracted by the holes, resulting in the main crack deflection.

Finally, let us highlight once more that simulations have also been conducted in the presence of heterogeneities consisting of a stiffer material. While a crack is generally attracted by the presence of a hole, the presence of stiffer inclusions tends to repel the crack. Besides, the presence of a heterogeneity consisting of the same elastic properties but with a different fracture energy is not able to influence a crack unless it is lo- cated on its path, contrary to heterogeneities consisting of different elastic properties.

\section{Conclusions and perspectives}

This work has investigated the capacities of the phasefield approach to reproduce specific features of dynamic crack propagation in brittle media. We have focused on the physical aspects which can be reproduced by numerical simulations using such a method. We considered a pre-strained PMMA plate configuration in which the initially stored energy is well defined and which leads to a progressive acceleration of the crack before reaching a steady-state regime. By removing the effect of stress waves induced by a suddenly applied loading in other numerical benchmark, we are able to better understand different aspects of dynamic crack propagation and the onset of branching. More precisely, various key results can be retained from this work:

- Crack propagation is characterized by a progressive widening of the damaged band width. It does not correspond to a later evolution of the crack tail due to delayed diffusion but occurs simultaneously to the crack tip advance. Besides, this wider band does not correspond to a larger regularization length but to a fully damaged zone with $d=1$ with increasing width, the transition zone from $d=1$ to $d=0$ remaining similar to the $1 \mathrm{D}$ solution profile.

- The damage band widening is associated with an increase of the apparent fracture energy $\Gamma$. A welldefined master-curve relating $\Gamma$ to the crack velocity in a single crack propagation phase is obtained. This relation does not depend on material properties but seems geometry-dependent.

- Macroscopic branching is observed when $\Gamma$ reaches a sufficiently high value, corresponding approximately to $2 G_{c}$. This observation favours an energetic criterion for branching. To our knowledge, this has not been remarked in previous works and would, therefore, require further investigations, both from a numerical and experimental point of view.

- A limiting velocity around $0.7 c_{R}$ is observed, which is in accordance with experimental results for this specific configuration. Computations in constrained propagation along a weakened interface enabled to reach velocities close to $c_{R}$, which has also been observed in previous experiments.

- The considered phase-field approach is able to naturally account for velocity-toughening both at a macroscale with damage band thickening and branching and at a smaller scale when interacting with heterogeneities through microbranching. 


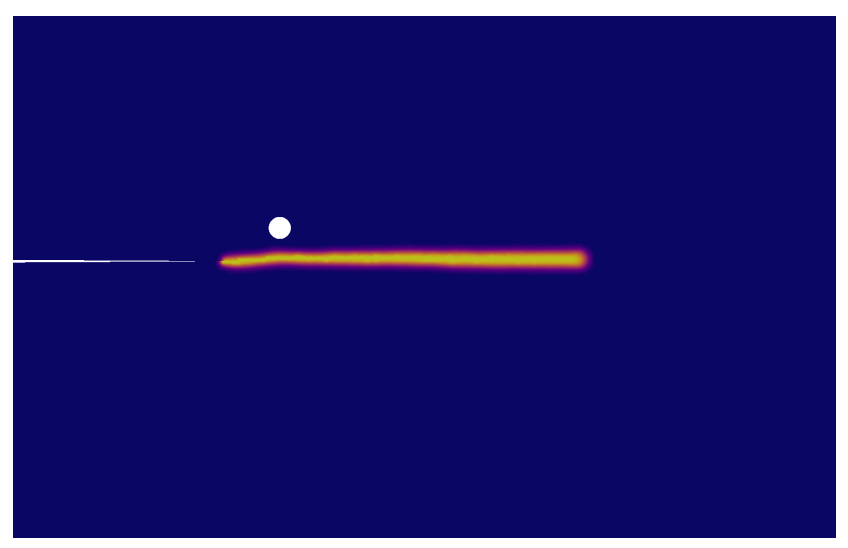

(a)

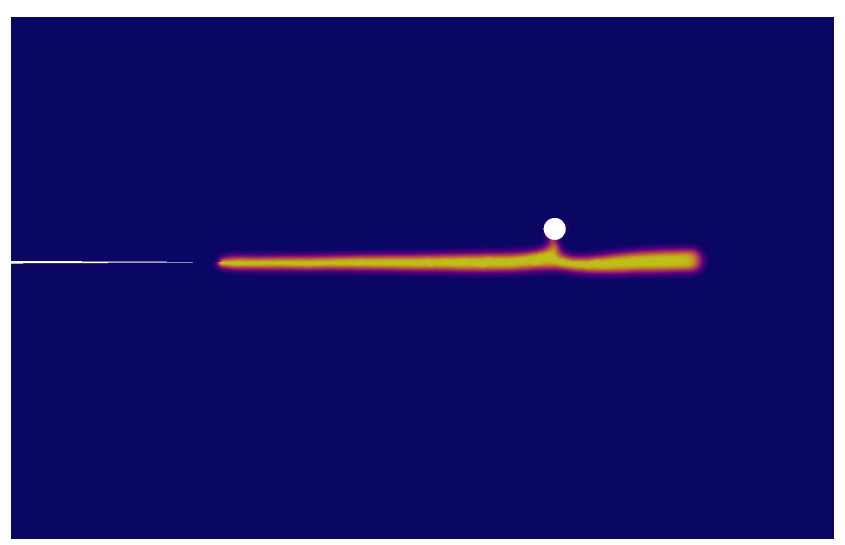

(b)

Fig. 18 Close-up view of the interaction of a crack with a single hole at $1 \mathrm{~mm}$ (a) and $6 \mathrm{~mm}$ (b) from the pre-notch tip with an offset of $0.6 \mathrm{~mm}$ from the middle plane $(\Delta U=0.04 \mathrm{~mm})$.

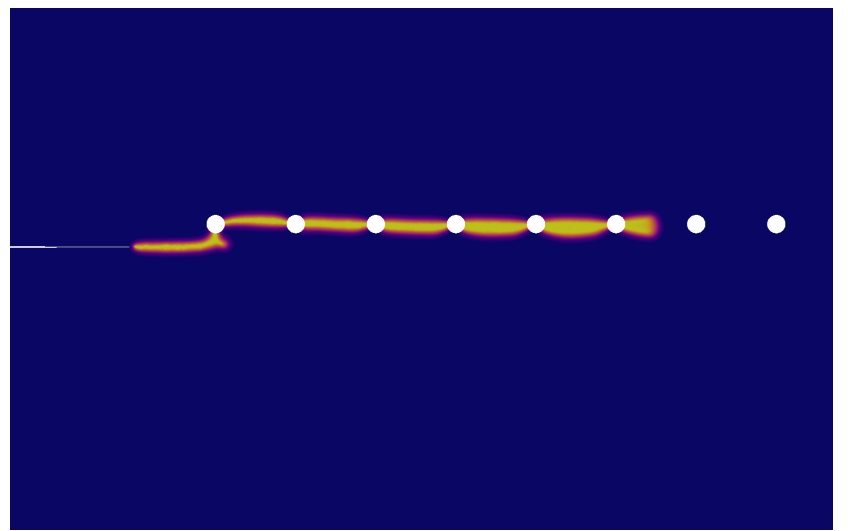

(a) $0.5 \mathrm{~mm}$ offset and $\Delta U=0.04 \mathrm{~mm}$

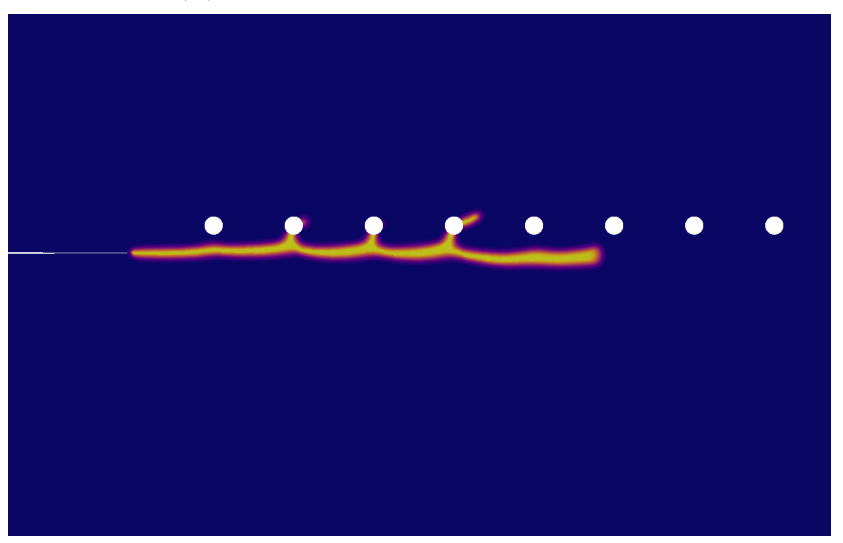

(c) $0.6 \mathrm{~mm}$ offset and $\Delta U=0.04 \mathrm{~mm}$

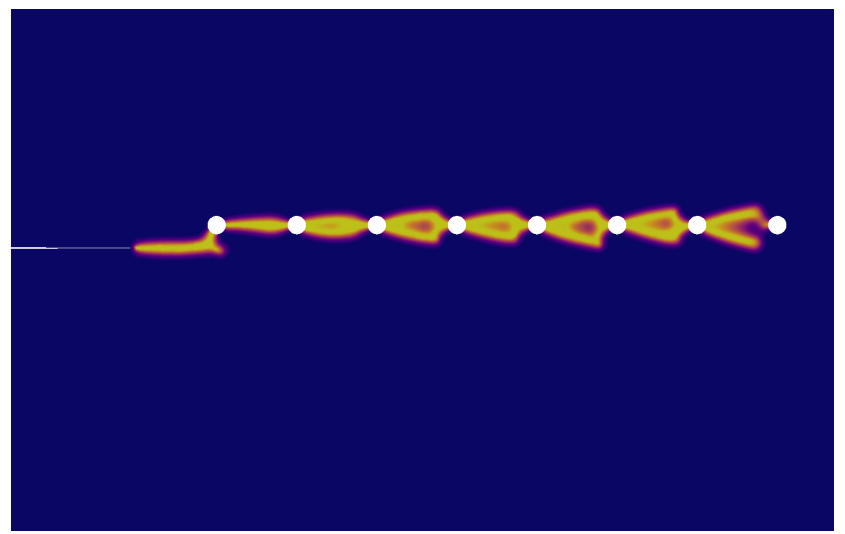

(b) $0.5 \mathrm{~mm}$ offset and $\Delta U=0.05 \mathrm{~mm}$

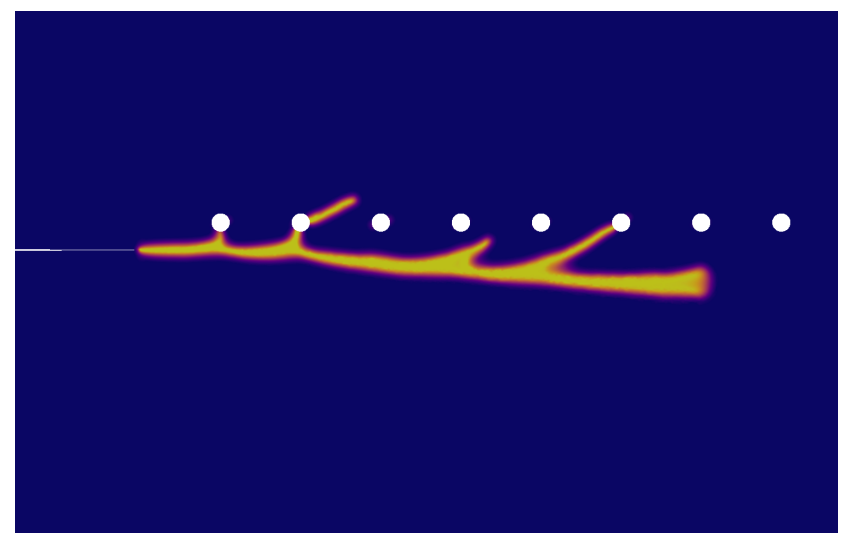

(d) $0.6 \mathrm{~mm}$ offset and $\Delta U=0.05 \mathrm{~mm}$

Fig. 19 Close-up view of the interaction of a crack with an array of holes located $0.5 \mathrm{~mm}((\mathrm{a})$ and (b)) or $0.6 \mathrm{~mm}((\mathrm{c})$ and (d)) away from the middle plane for two different loadings: $\Delta U=0.04 \mathrm{~mm}$ ((a) and (c)) or $\Delta U=0.05 \mathrm{~mm}((\mathrm{~b})$ and (d)). See also Movies $2-5$ in supplementary material. 
- Computations in presence of heterogeneities clearly showed the influence of defects on the crack propagation dynamics. In particular, holes on the crack path can delay macroscopic branching and lead to a stronger increase of the damage dissipation rate. Besides, the interaction of a crack with distant heterogeneities show that complex configurations can be obtained depending on the crack velocity and defect locations.

Regarding the last point, these preliminary simulations in heterogeneous media reveal a complex interplay between crack dynamics, material heterogeneities, microbranching and increase of fracture surface. The prediction of an effective toughness in dynamics seems to be a challenging question as various dissipative process can lead to a value higher than a simple average toughness. This question will require further investigation to, eventually, pave the way to the design of more efficient materials regarding dynamic fracture.

We believe that this work enabled to show that the phase-field approach is a legitimate candidate to study complex mechanisms of dynamic fracture. However, numerous questions remain still open. First, the pertinence of an energetically-based branching criterion should be more precisely assessed. Secondly, the present work did not manage to reproduce crack velocities obtained from the pre-strained PMMA plate experiments with the corresponding loading levels. Other models showed the same deficiencies and suggest rate effects have to be taken into account for PMMA. Finally, it is necessary to investigate the influence of $3 \mathrm{D}$ effects and the role of random material heterogeneities to better reproduce such experimental results.

Acknowledgements The authors would like to acknowledge Corrado Maurini and Li Tianyi for sharing FEniCSbased implementation of damage-gradient models.

\section{References}

1. Ambati, M., Gerasimov, T., De Lorenzis, L.: Phase-field modeling of ductile fracture. Computational Mechanics 55(5), 1017-1040 (2015)

2. Ambrosio, L., Tortorelli, V.M.: Approximation of functional depending on jumps by elliptic functional via tconvergence. Communications on Pure and Applied Mathematics 43(8), 999-1036 (1990)

3. Amor, H., Marigo, J.J., Maurini, C.: Regularized formulation of the variational brittle fracture with unilateral contact: Numerical experiments. Journal of the Mechanics and Physics of Solids 57(8), 1209-1229 (2009)

4. Balay, S., Abhyankar, S., Adams, M.F., Brown, J., Brune, P., Buschelman, K., Dalcin, L., Eijkhout, V., Gropp, W.D., Kaushik, D., Knepley, M.G., McInnes, L.C., Rupp,
K., Smith, B.F., Zampini, S., Zhang, H., Zhang, H.: PETSc Web page. http://www.mcs.anl.gov/petsc (2016)

5. Barenblatt, G.I.: The mathematical theory of equilibrium cracks in brittle fracture. Advances in applied mechanics 7, 55-129 (1962)

6. Belytschko, T., Chen, H., Xu, J., Zi, G.: Dynamic crack propagation based on loss of hyperbolicity and a new discontinuous enrichment. International Journal for Numerical Methods in Engineering 58(12), 1873-1905 (2003)

7. Bobaru, F., Zhang, G.: Why do cracks branch? A peridynamic investigation of dynamic brittle fracture. International Journal of Fracture 196(1-2), 59-98 (2015)

8. Borden, M.J., Verhoosel, C.V., Scott, M.A., Hughes, T.J., Landis, C.M.: A phase-field description of dynamic brittle fracture. Computer Methods in Applied Mechanics and Engineering 217, 77-95 (2012)

9. Bouchbinder, E., Goldman, T., Fineberg, J.: The dynamics of rapid fracture: instabilities, nonlinearities and length scales. Reports on Progress in Physics 77(4), 046,501 (2014)

10. Bouchbinder, E., Mathiesen, J., Procaccia, I.: Branching instabilities in rapid fracture: Dynamics and geometry. Physical Review E 71(5), 056,118 (2005)

11. Bourdin, B., Francfort, G.A., Marigo, J.J.: Numerical experiments in revisited brittle fracture. Journal of the Mechanics and Physics of Solids 48(4), 797-826 (2000)

12. Bourdin, B., Francfort, G.A., Marigo, J.J.: The variational approach to fracture. Journal of elasticity 91(1-3), 5-148 (2008)

13. Bourdin, B., Larsen, C.J., Richardson, C.L.: A timediscrete model for dynamic fracture based on crack regularization. International Journal of Fracture 168(2), 133$143(2011)$

14. Broberg, K.: How fast can a crack go? Materials Science 32(1), 80-86 (1996)

15. Cazes, F., Moës, N.: Comparison of a phase-field model and of a thick level set model for brittle and quasi-brittle fracture. International Journal for Numerical Methods in Engineering 103(2), 114-143 (2015)

16. Dalmas, D., Guerra, C., Scheibert, J., Bonamy, D.: Damage mechanisms in the dynamic fracture of nominally brittle polymers. International Journal of Fracture 184(1), 93-111 (2013)

17. Dugdale, D.S.: Yielding of steel sheets containing slits. Journal of the Mechanics and Physics of Solids 8(2), 100$104(1960)$

18. Falk, M.L., Needleman, A., Rice, J.R.: A critical evaluation of cohesive zone models of dynamic fracture. Le Journal de Physique IV 11(PR5), Pr5-43 (2001)

19. Fineberg, J., Marder, M.: Instability in dynamic fracture. Physics Reports 313(1), 1-108 (1999)

20. Fisher, D.S., Dahmen, K., Ramanathan, S., Ben-Zion, Y.: Statistics of earthquakes in simple models of heterogeneous faults. Physical Review Letters 78(25), 4885 (1997)

21. Francfort, G.A., Marigo, J.J.: Revisiting brittle fracture as an energy minimization problem. Journal of the Mechanics and Physics of Solids 46(8), 1319-1342 (1998)

22. Freund, L.B.: Dynamic fracture mechanics. Cambridge university press (1998)

23. Gao, H.: Surface roughening and branching instabilities in dynamic fracture. Journal of the Mechanics and Physics of Solids 41(3), 457-486 (1993)

24. Goldman, T., Cohen, G., Fineberg, J.: Origin of the microbranching instability in rapid cracks. Physical Review Letters 114(5), 054,301 (2015) 
25. Guerra, C., Scheibert, J., Bonamy, D., Dalmas, D.: Understanding fast macroscale fracture from microcrack post mortem patterns. Proceedings of the National Academy of Sciences 109(2), 390-394 (2012)

26. Guerra Amaro, C.M.: Dynamic fracture in brittle amorphous materials : Dissipation mechanisms and dynamically-induced microcracking in PMMA. Theses, Ecole Polytechnique (2009)

27. Henry, H.: Study of the branching instability using a phase field model of inplane crack propagation. EPL (Europhysics Letters) $\mathbf{8 3}(1), 16,004$ (2008)

28. Henry, H., Adda-Bedia, M.: Fractographic aspects of crack branching instability using a phase-field model. Physical Review E 88(6), 060,401 (2013)

29. Hofacker, M., Miehe, C.: Continuum phase field modeling of dynamic fracture: variational principles and staggered FE implementation. International Journal of Fracture 178(1-2), 113-129 (2012)

30. Hofacker, M., Miehe, C.: A phase field model of dynamic fracture: Robust field updates for the analysis of complex crack patterns. International Journal for Numerical Methods in Engineering 93(3), 276-301 (2013)

31. Jirasek, M.: Nonlocal models for damage and fracture: comparison of approaches. International Journal of Solids and Structures 35(31), 4133-4145 (1998)

32. Johnson, E.: Process region changes for rapidly propagating cracks. International Journal of Fracture 55(1), 47-63 (1992)

33. Karma, A., Kessler, D.A., Levine, H.: Phase-field model of mode III dynamic fracture. Physical Review Letters 87(4), 045,501 (2001)

34. Karma, A., Lobkovsky, A.E.: Unsteady crack motion and branching in a phase-field model of brittle fracture. Physical Review Letters 92(24), 245,510 (2004)

35. Lapusta, N., Liu, Y.: Three-dimensional boundary integral modeling of spontaneous earthquake sequences and aseismic slip. Journal of Geophysical Research: Solid Earth 114(B9), n/a-n/a (2009)

36. Li, T., Marigo, J.J., Guilbaud, D., Potapov, S.: Variational approach to dynamic brittle fracture via gradient damage models. In: Applied Mechanics and Materials, vol. 784, pp. 334-341. Trans Tech Publ (2015)

37. Li, T., Marigo, J.J., Guilbaud, D., Potapov, S.: Gradient damage modeling of brittle fracture in an explicit dynamics context. International Journal for Numerical Methods in Engineering in press, $\mathrm{n} / \mathrm{a}-\mathrm{n} / \mathrm{a}$ (2016). Nme.5262

38. Li, T., Maurini, C.: FEniCS (Dynamic) Gradient Damage. https://bitbucket.org/litianyi/dynamic-gradientdamage (2015)

39. Livne, A., Bouchbinder, E., Fineberg, J.: Breakdown of linear elastic fracture mechanics near the tip of a rapid crack. Physical Review Letters 101(26), 264,301 (2008)

40. Logg, A., Mardal, K.A., Wells, G.: Automated solution of differential equations by the finite element method: The FEniCS book, vol. 84. Springer Science \& Business Media (2012)

41. Lorentz, E., Cuvilliez, S., Kazymyrenko, K.: Modelling large crack propagation: from gradient damage to cohesive zone models. International Journal of Fracture 178(1-2), 85-95 (2012)

42. Marder, M.: New dynamical equation for cracks. Physical Review Letters 66(19), 2484 (1991)

43. May, S., Vignollet, J., De Borst, R.: A numerical assessment of phase-field models for brittle and cohesive fracture: $\Gamma$-convergence and stress oscillations. European Journal of Mechanics-A/Solids 52, 72-84 (2015)
44. Miehe, C., Hofacker, M., Welschinger, F.: A phase field model for rate-independent crack propagation: Robust algorithmic implementation based on operator splits. Computer Methods in Applied Mechanics and Engineering 199(45), 2765-2778 (2010)

45. Miehe, C., Welschinger, F., Hofacker, M.: Thermodynamically consistent phase-field models of fracture: Variational principles and multi-field FE implementations. International Journal for Numerical Methods in Engineering 83(10), 1273-1311 (2010)

46. Moës, N., Dolbow, J., Belytschko, T.: A finite element method for crack growth without remeshing. International Journal for Numerical Methods in Engineering 46(1), 131-150 (1999)

47. Moës, N., Stolz, C., Bernard, P.E., Chevaugeon, N.: A level set based model for damage growth: the thick level set approach. International Journal for Numerical Methods in Engineering 86(3), 358-380 (2011)

48. Murali, P., Guo, T., Zhang, Y., Narasimhan, R., Li, Y., Gao, H.: Atomic scale fluctuations govern brittle fracture and cavitation behavior in metallic glasses. Physical Review Letters 107(21), 215,501 (2011)

49. Pandolfi, A., Ortiz, M.: An eigenerosion approach to brittle fracture. International Journal for Numerical Methods in Engineering 92(8), 694-714 (2012)

50. Patinet, S., Vandembroucq, D., Roux, S.: Quantitative prediction of effective toughness at random heterogeneous interfaces. Physical Review Letters 110(16), 165,507 (2013)

51. Peerlings, R., De Borst, R., Brekelmans, W., Geers, M.: Gradient-enhanced damage modelling of concrete fracture. Mechanics of Cohesive-frictional Materials 3(4), 323-342 (1998)

52. Pham, K., Amor, H., Marigo, J.J., Maurini, C.: Gradient damage models and their use to approximate brittle fracture. International Journal of Damage Mechanics 20(4), 618-652 (2011)

53. Pijaudier-Cabot, G., Bazant, Z.P.: Nonlocal damage theory. Journal of engineering mechanics 113(10), 15121533 (1987)

54. Ravi-Chandar, K.: Dynamic fracture. Elsevier (2004)

55. Ravi-Chandar, K., Knauss, W.: An experimental investigation into dynamic fracture: II. Microstructural aspects. International Journal of Fracture 26(1), 65-80 (1984)

56. Ravi-Chandar, K., Knauss, W.: An experimental investigation into dynamic fracture: III. On steady-state crack propagation and crack branching. International Journal of Fracture 26(2), 141-154 (1984)

57. Ravi-Chandar, K., Yang, B.: On the role of microcracks in the dynamic fracture of brittle materials. Journal of the Mechanics and Physics of Solids 45(4), 535-563 (1997)

58. Rice, J.R.: Physical Aspects of Fracture, chap. Some Studies of Crack Dynamics, pp. 3-11. Springer Netherlands, Dordrecht (2001)

59. Seelig, T., Gross, D.: On the interaction and branching of fast running cracksa numerical investigation. Journal of the Mechanics and Physics of Solids 47(4), 935-952 (1999)

60. Sharon, E., Gross, S.P., Fineberg, J.: Energy dissipation in dynamic fracture. Physical Review Letters 76(12), 2117 (1996)

61. Sicsic, P., Marigo, J.J.: From gradient damage laws to Griffiths theory of crack propagation. Journal of Elasticity 113(1), 55-74 (2013)

62. Stolarska, M., Chopp, D., Moës, N., Belytschko, T.: Modelling crack growth by level sets in the extended finite element method. International journal for numerical methods in Engineering 51(8), 943-960 (2001) 
63. Stroh, A.N.: A theory of the fracture of metals. Advances in Physics 6(24), 418-465 (1957)

64. Vasoya, M., Unni, A.B., Leblond, J.B., Lazarus, V., Ponson, L.: Finite size and geometrical non-linear effects during crack pinning by heterogeneities: An analytical and experimental study. Journal of the Mechanics and Physics of Solids 89, $211-230$ (2016)

65. Verhoosel, C.V., Borst, R.: A phase-field model for cohesive fracture. International Journal for Numerical Methods in Engineering 96(1), 43-62 (2013)

66. Washabaugh, P.D., Knauss, W.: A reconciliation of dynamic crack velocity and Rayleigh wave speed in isotropic brittle solids. International Journal of Fracture 65(2), 97-114 (1994)

67. Wolff, C., Richart, N., Molinari, J.F.: A non-local continuum damage approach to model dynamic crack branching. International Journal for Numerical Methods in Engineering 101(12), 933-949 (2015)

68. Xu, D., Liu, Z., Liu, X., Zeng, Q., Zhuang, Z.: Modeling of dynamic crack branching by enhanced extended finite element method. Computational Mechanics 54(2), 489$502(2014)$

69. Xu, X.P., Needleman, A.: Numerical simulations of fast crack growth in brittle solids. Journal of the Mechanics and Physics of Solids 42(9), 1397-1434 (1994)

70. Yoffe, E.H.: The moving Griffith crack. The London, Edinburgh, and Dublin Philosophical Magazine and Journal of Science 42(330), 739-750 (1951)

71. Yu, C., Pandolfi, A., Ortiz, M., Coker, D., Rosakis, A.: Three-dimensional modeling of intersonic shear-crack growth in asymmetrically loaded unidirectional composite plates. International Journal of Solids and Structures 39(25), 6135-6157 (2002)

72. Zhou, F.: Study on the macroscopic behavior and the microscopic process of dynamic crack propagation. Ph.D. thesis, PhD dissertation. The University of Tokyo, Tokyo (1996)

73. Zhou, F., Molinari, J.F., Shioya, T.: A rate-dependent cohesive model for simulating dynamic crack propagation in brittle materials. Engineering Fracture Mechanics 72(9), 1383-1410 (2005) 